\title{
No Good Deals - No Bad Models
}

\author{
Nina Boyarchenko ${ }^{1}$, Mario Cerrato ${ }^{2}$, John Crosby ${ }^{2,3}$, and Stewart Hodges ${ }^{4}$ \\ ${ }^{1}$ Federal Reserve Bank of New York \\ ${ }^{2}$ Centre for Economic and Financial Studies, University of Glasgow and \\ Glasgow University Adam Smith Business School \\ ${ }^{3}$ Grizzly Bear Capital \\ ${ }^{4}$ Cass Business School, City University, London
}

January 10, 2013

\begin{abstract}
Faced with the problem of pricing complex contingent claims, an investor seeks to make his valuations robust to model uncertainty. We construct a notion of a modeluncertainty-induced utility function and show that model uncertainty increases the investor's effective risk aversion. Using the model-uncertainty-induced utility function, we extend the "No Good Deals" methodology of Cochrane and Saá-Requejo [2000] to compute lower and upper good deal bounds in the presence of model uncertainty. We illustrate the methodology using some numerical examples.
\end{abstract}

Keywords: Asset pricing theory; Good deal bounds; Knightian uncertainty; Model uncertainty; Contingent claim pricing. model-uncertainty-induced utility function JEL classification codes: G12, G13.

Boyarchenko: nina.boyarchenko@ny.frb.org; Cerrato: Mario.Cerrato@glasgow.ac.uk; Crosby: johnc2205@yahoo.com; Hodges: stewart.hodges.1@city.ac.uk. The views presented here are the authors' and are not representative of the views of the Federal Reserve Bank of New York or of the Federal Reserve System.

Acknowledgements: We are indebted to Volker Bosserhoff for excellent research assistance. We thank Craig Burnside, Javier Gil-Bazo and seminar participants at the Barcelona Graduate School of Economics for comments. Any remaining errors are the authors'. 


\section{Introduction}

The recent financial crisis has highlighted the significance of unhedgable, illiquid positions for individual financial institutions and for the global financial system as a whole. Indeed, the Basel Committee on Banking Supervision notes that

One of the key lessons of the crisis has been the need to strengthen the risk coverage of the capital framework. Failure to capture major on-and off-balance sheet risks, as well as derivative related exposures, was a key destabilising factor during the crisis. ${ }^{1}$

While concepts like marking-to-market and risk weighting of individual positions are incorporated in all three pillars of the Basel III capital regulation, the academic literature has lagged in providing a robust, model-free way of implementing these in practice. In this paper, we propose a methodology to compute lower and upper bounds on prices of complex (potentially non-traded) securities that is robust to misspecifications of the model of the underlying cash-flows.

Our methodology incorporates a concern for robustness to model uncertainty into the "No Good Deals" methodology of Cochrane and Saá-Requejo [2000]. The no good deals methodology refines the lower and upper arbitrage bounds on securities prices by imposing a maximal admissible Sharpe ratio for trading strategies: Just as arbitrages are ruled out for giving investors a free lunch, so very high Sharpe ratios are ruled out on the grounds that, if allowed, a very high Sharpe ratio would represent such a good deal that (Ross [1976]) it should not exist in equilibrium. In this paper, we extend the intuition of Cochrane and SaáRequejo [2000] and argue that investors should also be restricted in the set of models they are allowed to use in computing the maximal Sharpe ratio. In particular, we assume that investors evaluate alternative trading strategies using Hansen and Sargent [2008] multiplier preferences. While an investor might have an estimate of the evolution of the underlying

\footnotetext{
${ }^{1}$ Source: Basel III.
} 
shocks in an economy, she recognizes that her estimate may not be the true data-generating process. Thus, she considers a set of alternative models, with her preference for robustness forcing her to choose the "worst-case" model in computing the good deal bounds.

Model uncertainty impacts the good deal bounds in two ways. First, since the uncertainty averse agent assigns higher probabilities (than under the approximating model) to states with lower payoffs (or higher losses), the good deal bounds are wider in the presence of model uncertainty than in its absence. Intuitively, the lower bound on the price of the security is the bid that an agent buying the security is willing to submit; in the presence of model uncertainty, a misspecification averse agent fears that the security is less valuable than the traditional good deal bounds would suggest. Similarly, the upper bound is set by the ask price of the seller; in the presence of model uncertainty, a misspecification averse agent fears that the security is more valuable. Second, while the right to dynamically hedge is always a valuable one, we show that model uncertainty reduces the benefit of dynamic hedging relative to static hedging (see Carr, Ellis, and Gupta [1998] for a discussion of static hedging strategies for complex options). In particular, as the investor becomes more averse to model uncertainty, the good deal bounds converge to the arbitrage bounds and the dynamic hedging strategy converges to the static one.

We build on the results of Černý [2003] - that the bound on the volatility of the pricing kernel imposed by Cochrane and Saá-Requejo [2000] in their derivation of good deal bounds is intimately tied with quadratic utility - to derive the analogous restrictions on the pricing kernel in the presence of model uncertainty. More specifically, we begin by introducing the notion of a model-uncertainty-induced utility function. The concern for model misspecification increases the effective risk aversion of the investor, leading to wider good deal bounds. Using the implied restrictions on the pricing kernel for quadratic, exponential and CRRA utility functions, we formulate the no good deal problem in the presence of model uncertainty and solve for the lower and upper bounds. Finally, we show how to estimate the degree of 
investors' aversion to model uncertainty, and illustrate the method using some numerical examples.

\section{Literature Review}

Our framework builds upon that of Cochrane and Saá-Requejo [2000]. Bernardo and Ledoit [2000] simultaneously developed an alternative framework for constructing good deal bounds which uses gain-loss ratios rather than Sharpe ratios. Černý [2003] and Černý and Hodges [2001] explain how the use of gain-loss ratios puts the Bernardo and Ledoit [2000] framework into a rather different category compared to the Sharpe ratio and generalised Sharpe ratio based framework and, for this reason, we don't consider it further. There have been some follow-up papers to the work of Cochrane and Saá-Requejo [2000] - Hodges [2009] is a comprehensive review - but as he points out, most of them are highly mathematical and the economic intuition of Cochrane and Saá-Requejo [2000], and of Hansen and Jagannathan [1991], and its potential use as a practical tool for practitioners and regulators alike has been obscured.

The seminal contribution of the "No Good Deals" methodology of Cochrane and SaáRequejo [2000] is to narrow the arbitrage bounds by additionally requiring that the volatility of the pricing kernel is bounded which, in view of Hansen and Jagannathan [1991], is the same as bounding the maximum available Sharpe ratio. The resulting good deal bounds both rule out arbitrage and rule out the possibility of forming a portfolio of the complex security (termed the focus asset) and of a set of hedging assets (termed basis assets) which has more than some given Sharpe ratio. Just as arbitrages are ruled out for giving investors a free lunch, so very high Sharpe ratios are ruled out on the grounds that, if allowed, a very high Sharpe ratio would represent such a good deal that (Ross [1976]) it should not exist in equilibrium. Hence, "No Good Deals" introduce a partial equilibrium consideration into the pricing of complex securities but, crucially, without having to make any strong assumptions (for example, the precise specification of investors' utility functions) concerning the nature of 
the equilibrium. This is especially pertinent to the pricing and trading of complex securities where agents (typically employees of investment banks or hedge funds) are rarely acting on their own account and hence personalised measures of preferences such as a utility function (even if estimable) may be inappropriate.

Sharpe ratios de-personalise the selection of a criterion to narrow the arbitrage bounds. If an investor (or, more generally, a financial institution) would be prepared to enter into any trade that is either an arbitrage or that is expected to deliver more than a specified Sharpe ratio, then she will, in all likelihood, be prepared to trade a complex security priced by the same criterion. Further, Sharpe ratios are simple and widely used so it is likely that there will be other investors (or financial institutions) who would be prepared to trade on the same terms and who would therefore be prepared to take the other side of the trade should the first investor decide to liquidate her position. Hence the use of Sharpe ratios gives a market (as opposed to an individualistic) perspective.

Although the no good deals methodology provides a compelling and economically-motivated way for investors to consider the impact of unhedgeable market risks on the prices of complex securities, the recent history of financial institutions suggest that these considerations are insufficient to incentivize market participants to be sufficiently conservative in their valuations. Even before the global financial crisis of 2007-2009, there had been a series of financial institutions (such as Bank of Tokyo/Mitsusbishi in 1997, Nat-West in 1997, Bankers Trust in 1998, Amaranth Advisors LLC in 2006) reporting large losses on their (supposedly, hedged) positions in over-the-counter (OTC) derivatives. The losses have continued after the crisis. For example, in April 2011, Reuters reported that Mitsubishi UFJ Morgan Stanley Securities (a joint venture between a Japanese bank and Morgan Stanley) had incurred losses of more than 1.75 billion dollars on its positions in complex derivatives. Furthermore, these losses were not attributable to the earthquake and tsunami which had struck Japan six weeks earlier. Instead, Reuters directly quoted a senior official at the joint venture as saying that the losses had accumulated (and we quote verbatim) "bit by bit" over a period of months. 
Whilst the exact details of how these losses arose is still unclear, the quotation suggests that the positions were incorrectly marked-to-market or suffered from gradual hedge-slippage as the prices of hedging assets diverged from their model predictions.

The aftermath of the global financial crisis has intensified political and regulatory scrutiny of the hedging and risk-management of OTC derivatives. Although both the new Basel III banking regulation as well as its predecessor Basel II specifically require banks to take into account model risk or uncertainty, based on our conversations with traders at leading investment banks, there does not seem to any consistent methodology to doing so. Some banks do not follow the framework in this regard due to auditor requirements on mark-tomarket accounting, while others have ad-hoc valuation adjustments to their mark-to-market prices for some types of securities but not for others. This may be due to a lack of a consensus on how to generate mark-to-market or reservation prices, or it may be because traders and senior executives are incentivized by bonus structures to maximize up-front profits and not to place conservative reservation prices on their positions, especially for complex securities with long maturities. Either way, our paper suggests a way forward by requiring investors to incorporate a concern for model misspecification in their price estimates.

Model uncertainty has the additional feature of reflecting a different dimension of uncertainty faced by market participants. The original insight of Gilboa and Schmeidler [1989] that Knightian (or model) uncertainty can explain the Ellsberg [1961] paradox - has more recently been extended by Hansen and Sargent [2008], Hansen, Sargent, and Tallarini [1999], Hansen and Sargent [2001, 1995], Cagetti, Hansen, Sargent, and Williams [2002], Barillas, Hansen, and Sargent [2009], Maenhout [2004], Garlappi, Uppal, and Wang [2007] and Uppal and Wang [2003] to show that investors have a fundamentally different aversion to model uncertainty. For example, Barillas et al. [2009] and Maenhout [2004] show that the classical equity premium puzzle of Mehra and Prescott [1985] can be resolved by allowing agents to have robustness preferences over alternative models; Uppal and Wang [2003] show that model uncertainty can also be used to explain the home-bias puzzle of Cooper and Kaplanis 
[1994], Coval and Moskowitz [1999] and Huberman [2001]. Additional studies examining the implications of model uncertainty for equities and non-defaultable bonds include Anderson, Ghysels, and Juergens [2009], Bossaerts, Ghirardato, Guarnaschelli, and Zame [2010], Leippold, Trojani, and Vanini [2008], Cao, Wang, and Zhang [2005], Boyle, Garlappi, Uppal, and Wang [2012] and Cvitanic, Lazrak, Martellini, and Zapatero [2011]. Furthermore, recent studies have demonstrated the importance of model uncertainty in modeling the prices of complex securities. Liu, Pan, and Wang [2005] find that aversion to model uncertainty plays an important role in explaining the pricing differentials among options on the S \& P 500 stock index across moneyness (that is, the "smirk" or "smile" seen in implied volatilities). Using a similar intuition, Drechsler [2012] finds that concerns for model misspecification explain the large premia in index options. Finally, Boyarchenko [2012] argues that model uncertainty can explain the behavior of credit swap spreads (CDS) on financial institutions during the recent financial crisis.

While the implications for asset pricing and portfolio choice under model uncertainty have been extensively considered, incorporating model uncertainty into the pricing of contingent claims has been less well-developed. Boyle, Feng, Tian, and Wang [2008] argue that, in incomplete markets, the multiplicity of available stochastic factors naturally leads to a concern for model misspecification. Instead of following the literature on robust preferences, however, they choose the optimal pricing kernel to limit the variation in the price of a contingent claim when the underlying asset's payoff is slightly perturbed. The main advantage of this approach is to construct perturbations that are based on the volatility of the basis asset, which is the pertinent quantity in pricing options on the asset. This natural link comes at a cost, however, with the Black and Scholes [1973] model used as the benchmark model. Furthermore, the authors focus on standard European options. While they briefly discuss the pricing of other types of derivatives, the continuous time setup makes these extensions non-trivial for the case of path-dependent or early exercise (American) options. 
In contrast, we make the lower and upper bounds on the value of a contingent claim robust to model uncertainty by choosing the pricing kernel subject to a relative entropy criterion. Thus, our perturbations are based on the conditional entropy of the pricing kernel, allowing for non-Gaussian distributions even in the static case. An additional advantage of our approach is in considering a discrete-time, discrete space (lattice) formulation. The lattice approach simplifies the pricing of finite horizon, path-dependent options while emphasizing their illiquid nature. Further, any continuous space stochastic process can be well approximated by a discrete lattice, provided that the lattice has sufficient states. Finally, continuous trading is not always possible in real markets; the discrete-time formulation naturally incorporates these breaks in trading activity.

The rest of this paper is structured as follows: Section 2 provides an overview of the "No Good Deals" methodology. Section 3 introduces model uncertainty and shows how the Cochrane and Saá-Requejo [2000] bound $\mathbb{E}\left[m^{2}\right] \leq A^{2}$ should be modified in its presence. Section 4 defines and solves for the lower and upper good deal bounds. Section 5 shows how to estimate the parameter $(\Omega)$ that controls aversion to model uncertainty. Section 6 extends our methodology to multiple time periods. Section 7 provides some numerical results. Section 8 concludes. Proofs are relegated to the appendix.

\section{Good deal bounds without model uncertainty}

We briefly review the "No Good Deals" methodology of Cochrane and Saá-Requejo [2000] (in the absence of model uncertainty) and its subsequent extensions (Hodges [1998], Černý and Hodges [2001] and Černý [2003]) to alternative utility function settings. The starting point of this methodology is to describe the distribution of future payoffs to financial assets or outcomes of economic variables (such as interest-rates). This is equivalent to specifying a reference probability measure $\mathbb{P}$ over the possible states of the world. Although our focus in this paper is to allow agents to be uncertain about the probability measure $\mathbb{P}$, in this section 
we ignore model uncertainty concerns and take $\mathbb{P}$ as given. This allows us to outline the relationship between the "No Good Deals" methodology and expected utility maximization, making the link between model uncertainty and good deal bounds more immediate.

Consider pricing a complex security or contingent claim (or a portfolio of these), and assume that this focus asset (in the terminology of Cochrane and Saá-Requejo [2000]) pays $x_{c}$ at time 1 . In addition, assume that there are $N_{b}$ basis assets, traded in an active, liquid market. Each basis asset $i=1, \ldots, N_{b}$ pays $x_{i}$ at time 1 , with the time 0 market price given by $p_{i}$. We denote by $\mathbf{p}$ and $\mathbf{x}$ respectively the $N_{b}$ - dimensional vectors whose $i^{\text {th }}$ elements are $p_{i}$ and $x_{i}$, respectively. We assume that the agent in question does not know the pricing kernel used by the marginal investor in the market and instead uses the prices of the basis assets to inform herself about the possible pricing kernels in the economy. More specifically, a candidate pricing kernel $m$ must exactly price the basis assets under the reference probability measure $\mathbb{P}$, so that $\mathbb{E}^{\mathbb{P}}\left[m x_{i}\right]=p_{i}$, for each $i=1, \ldots, N_{b}$, and imply the absence of arbitrage opportunities, so that $m \geq 0 .^{2}$ Notice that, since we do not assume that markets are complete, there can be multiple candidate pricing kernels that satisfy these conditions.

The innovation of Cochrane and Saá-Requejo [2000] is to restrict the set of candidate pricing kernels by requiring that the variance of a candidate pricing kernel is bounded from above. In particular, the lower $\underline{C}^{\text {NoMU }}$ and upper $\bar{C}^{\text {NoMU }}$ good deal bounds on the time 0 price of the focus asset satisfy

$$
\underline{C}^{\mathrm{NoMU}}=\inf _{m}\left\{\mathbb{E}^{\mathbb{P}}\left[m x_{c}\right] \text { such that } \mathbb{E}^{\mathbb{P}}[m \mathbf{x}]=\mathbf{p}, m \geq 0, \mathbb{E}^{\mathbb{P}}\left[m^{2}\right] \leq A^{2}\right\}
$$

with the infimum replaced by a supremum for $\bar{C}^{\mathrm{NoMU}}$. The first two constraints enforce the exact repricing of the basis assets and absence of arbitrage. The final constraint bounds the variance of candidate pricing kernels and implies (see Hansen and Jagannathan [1991]) a maximal available Sharpe ratio. Notice that the bound on the variance of the candidate

\footnotetext{
${ }^{2}$ See e.g. Cochrane [2005].
} 
pricing kernel must be large enough to allow for exact pricing of the basis assets; in particular, we assume in this Section that $A^{2} \geq A^{\star N o M U 2}$ where

$$
A^{\star N o M U} 2=\inf _{m}\left\{\mathbb{E}^{\mathbb{P}}\left[m^{2}\right] \text { such that } \mathbb{E}^{\mathbb{P}}[m \mathbf{x}]=\mathbf{p}, m \geq 0\right\}
$$

To solve for the lower and upper good deal bounds, consider two cases: (1) $\mathbb{E}^{\mathbb{P}}\left[m^{2}\right] \leq A^{2}$ binds and (2) $\mathbb{E}^{\mathbb{P}}\left[m^{2}\right] \leq A^{2}$ is slack. ${ }^{3}$ Rearranging the solutions of Cochrane and Saá-Requejo [2000], we can express the lower and upper bounds in the two cases as follows.

Case (1) $\mathbb{E}^{\mathbb{P}}\left[m^{2}\right] \leq A^{2}$ binds:

$$
\begin{array}{ll}
\underline{C}^{\text {NoMU }} \text { solves: } & A^{2}=\max _{\mu>0, \mathbf{v}}\left\{2 \mathbf{v}^{\prime} \mathbf{p}-2 \mu \underline{C}^{\mathrm{NoMU}}+\mathbb{E}^{\mathbb{P}}\left[U^{T}\left(\mu x_{c}-\mathbf{v}^{\prime} \mathbf{x}\right)\right]\right\}, \\
\bar{C}^{\text {NoMU }} \text { solves: } & A^{2}=\max _{\mu<0, \mathbf{v}}\left\{2 \mathbf{v}^{\prime} \mathbf{p}-2 \mu \bar{C}^{\mathrm{NoMU}}+\mathbb{E}^{\mathbb{P}}\left[U^{T}\left(\mu x_{c}-\mathbf{v}^{\prime} \mathbf{x}\right)\right]\right\} .
\end{array}
$$

Case (2) $\mathbb{E}^{\mathbb{P}}\left[m^{2}\right] \leq A^{2}$ slack:

$$
\begin{aligned}
& \text { If } A^{2}>\max _{\mathbf{v}, \mu}\left\{2 \mathbf{v}^{\prime} \mathbf{p}-2 \mu C_{\mathrm{Arb}}+\mathbb{E}^{\mathbb{P}}\left[U^{T}\left(\mu x_{c}-\mathbf{v}^{\prime} \mathbf{x}\right)\right]\right\} \text {, then } \\
& \underline{C}^{\mathrm{NoMU}}\left(\bar{C}^{\mathrm{NoMU}}\right) \text { equals the lower (or upper) arbitrage bound } C_{\mathrm{Arb}} .
\end{aligned}
$$

In equations (3) to $(5), U^{T}(V) \equiv-(\max (-V, 0))^{2}, \mathbf{v}$ is a $N_{b}$ - dimensional vector and $\mu$ is a scalar. The maximizations are made over choices of $\mu$ and $\mathbf{v}$ which play the role of positions taken in the focus asset and in the basis assets. The restriction $\mu>0$ in (3) (respectively, $\mu<0$ in (4)) corresponds to taking a long position (a short position of size $|\mu|)$, at time 0 , at a price of $\underline{C}^{\mathrm{NoMU}}$ (respectively, $\bar{C}^{\mathrm{NoMU}}$ ) to solve for the lower (upper) good deal bound. Notice that we can reinterpret (3) to (5) in terms of utility maximization.

${ }^{3}$ Cochrane and Saá-Requejo [2000] consider three different cases: (a) $m \geq 0$ slack, $\mathbb{E}^{\mathbb{P}}\left[m^{2}\right] \leq A^{2}$ binds, (b) both bind and (c) $m \geq 0$ binds, $\mathbb{E}^{\mathbb{P}}\left[m^{2}\right] \leq A^{2}$ slack. It was convenient for Cochrane and Saá-Requejo [2000] to do so because case (a) yields an analytical solution. However, it suits our purpose to combine cases (a) and (b) into one because the analytical solution available in case (a) is a special case of the (numerical) solution in case (b). 
Consider, for example, the lower bound in the case when $\mathbb{E}^{\mathbb{P}}\left[m^{2}\right] \leq A^{2}$ binds. The investor allocates her time 0 wealth between the basis assets and a long position in the focus asset to maximize her expected utility at time 1 , subject to the time 0 budget constraint, and evaluates possible outcomes using a (truncated) quadratic utility function. More generally, the "No Good Deals" methodology computes lower and upper good deal bounds which (1) solve for a specified level $A^{2}$ of reward-for-risk when such a solution exists or (2) are the arbitrage bounds $C_{\mathrm{Arb}}$ when the maximum achievable reward-for-risk is insufficient to reach the specified level $A^{2}$.

The link between the bound on the volatility of the pricing kernel and (truncated) quadratic utility is a consequence of convex duality. ${ }^{4}$ Using this intuition, Černý [2003] derives restrictions on the pricing kernel corresponding to exponential and CRRA (including $\log$ ) utility functions. These restrictions can also be viewed as restrictions on the certainty equivalent associated with the relevant utility function or on the optimal level of expected utility. Furthermore, just like the restriction on the pricing kernel $\mathbb{E}^{\mathbb{P}}\left[m^{2}\right] \leq A^{2}$ is equivalent to a bound on the maximum Sharpe ratio in the economy, the restrictions that Cerný $[2003]^{5}$ derives for exponential, CRRA and log utility functions are equivalent to bounds on the maximum "generalized" Sharpe ratios.

To link restrictions on the pricing kernel with utility functions, Černý [2003] considers an investor endowed with wealth $V_{0}$ at time 0 . The investor, without a concern for model uncertainty, maximizes her expected utility of time 1 wealth $V$ subject to the time 0 budget constraint

$$
\sup _{V} \mathbb{E}^{\mathbb{P}}[U(V)] \quad \text { such that } \quad \mathbb{E}^{\mathbb{P}}[m V]=V_{0},
$$

where $U(V)$ is the investor's utility function. Černý [2003] introduces Lagrange multipliers to solve this problem for various utility functions. In particular, for truncated quadratic

\footnotetext{
${ }^{4}$ See Cox and Huang [1989] for one of the first applications in utility maximization problems.

${ }^{5}$ See also Hodges [1998], Černý and Hodges [2001]
} 
utility $U(V)=-(\max (\bar{V}-V, 0))^{2}$, the maximum achievable value of $\mathbb{E}^{\mathbb{P}}[U(V)]$, subject to the constraint $\mathbb{E}^{\mathbb{P}}[m V]=V_{0}$, is

$$
\sup _{V} \mathbb{E}^{\mathbb{P}}[U(V)]=-\frac{\left(\bar{V}-V_{0}\right)^{2}}{\mathbb{E}^{\mathbb{P}}\left[m^{2}\right]}=\frac{U\left(V_{0}\right)}{\mathbb{E}^{\mathbb{P}}\left[m^{2}\right]} \equiv U\left(V_{0}+\mathrm{CE}\right),
$$

with the certainty equivalent associated with truncated quadratic utility given by

$$
\mathrm{CE}=\left(\bar{V}-V_{0}\right)\left(1-\frac{1}{\sqrt{\mathbb{E}^{\mathbb{P}}\left[m^{2}\right]}}\right)
$$

Thus, the bound on the volatility of the pricing kernel implies a bound on the maximum achievable certainty equivalent

$$
\mathbb{E}^{\mathbb{P}}\left[m^{2}\right] \leq A^{2} \Longleftrightarrow \sup _{V} \mathbb{E}^{\mathbb{P}}[U(V)] \leq U\left(V_{0}\right) / A^{2} \Longleftrightarrow \mathrm{CE} \leq\left(\bar{V}-V_{0}\right)\left(1-1 / \sqrt{A^{2}}\right)
$$

This gives us a dual interpretation on "No Good Deals": "No Good Deals" can be seen either as ruling out Sharpe ratios which are too high or as ruling out too high levels of expected utility (or certainty equivalent) relative to $U\left(V_{0}\right)$ (or $V_{0}$ ). While the first interpretation has the advantage of being independent from the specification of a utility function for the investor in question, the second interpretation will allow us to connect good deal bounds with model uncertainty.

We conclude this section by summarizing the basic assumptions that we maintain throughout the paper. We assume that there are $S$ possible time 1 states of the world. We denote the probability, under $\mathbb{P}$, of attaining state $s$ by $\mathbb{P}(s)$, for each $s=1, \ldots, S$. Finally, we assume that

Assumption 1 (1) S, the number of possible states of the world at time 1, is finite,

(2) $\mathbb{P}(s)>0, \forall s=1, \ldots, S$, so that zero probability states have been pruned,

(3) the time 1 payoffs are finite in each state 
(4) there are no arbitrage opportunities amongst the basis assets, and

(5) redundant basis assets have been pruned.

\section{$3 \quad$ Introducing model uncertainty}

In this section, we introduce the notion of a model-uncertainty-induced utility function and, similarly to Černý [2003], derive the implied bounds on candidate pricing kernels. Throughout the rest of the paper, we assume

Assumption 2 (1) The utility over wealth, $U(V)$, is bounded above by a finite positive constant $^{6}$ which, without loss of generality, can be taken to be zero. Hence, $U(V)$ takes on negative values i.e. $U(V) \leq 0$, for all $V$.

(2) $U(V)$ is non-decreasing, continuous, concave and differentiable with $U^{\prime}(V) \geq 0, U^{\prime \prime}(V) \leq$ $0, \lim _{V \rightarrow-\infty} V / U(V)=0$ and $\lim _{V \rightarrow \infty} U^{\prime}(V)=0$.

For future use, we also introduce $\operatorname{CARA}(U(V))=-U^{\prime \prime}(V) / U^{\prime}(V)$ to be the coefficient of absolute risk aversion of $U(V)$.

\subsection{Model uncertainty}

We begin by describing the economic setting considered in this paper. As in the previous Section, denote by $\mathbb{P}$ the reference probability measure over the possible states of the world. While the optimizing agent knows the model $\mathbb{P}$ to be the best estimate of the data-generating process given the information at her disposal, she recognizes that the model is estimated from a finite data-set. Thus, she worries that the true model may be in a set of alternative models $\mathcal{P}$ that are difficult for her to reject empirically. The investor guards against model

\footnotetext{
${ }^{6}$ This precludes log utility since it is unbounded. However, it is satisfied by the three utility functions that we will be interested in: Quadratic, exponential and CRRA: $U(V)=\bar{\beta} \frac{V^{1-\gamma}}{1-\gamma}$, for (the empirically relevant) $\gamma>1$.
} 
uncertainty by considering asset allocations that are robust across the set of alternative models. As shown in Hansen and Sargent [2001, 2008, 1995], Hansen et al. [1999] and Anderson, Hansen, and Sargent [2003], this is equivalent to evaluating future prospects under the worst-case model in the alternative set.

More formally, we index a member $\mathbb{P}^{\xi}$ of the set of alternative models $\mathcal{P}$ by its RadonNikodym derivative $\xi=d \mathbb{P}^{\xi} / d \mathbb{P}$. Notice that $\xi$ captures the likelihood ratio between the two models, and that the reference model $\mathbb{P}$ corresponds to the case $\xi=1$. To keep the interpretation of a likelihood ratio, we assume that $\xi$ is strictly positive, $\xi>0$, and integrates to $1, \mathbb{E}^{\mathbb{P}}[\xi]=1$. The model uncertainty averse agent then solves

$$
\inf _{\mathbb{P}^{\xi} \in \mathcal{P}}\left\{\sup _{V}\left\{\mathbb{E}^{\mathbb{P}^{\xi}}[f(\xi) U(V)] \quad \text { such that } \quad \mathbb{E}^{\mathbb{P}^{\xi}}\left[m^{\xi} V\right]=V_{0}\right\}\right\}
$$

That is, the investor maximizes her expected utility of future wealth subject to the initial budget constraint, while expressing her model uncertainty and desire for robustness by evaluating her future prospects under the worst-case (minimizing) model within the set of alternatives. The multiplicative penalty $f(\xi)$ disciplines the agent's decision making and restricts the set of alternative measures. We assume that

$$
f(\xi) \equiv 1-\Omega \log \xi
$$

where the constant $\Omega$ satisfies $1 \leq \Omega<\infty$ and captures the degree of investor's aversion to model uncertainty. As $\Omega$ increases, the agent becomes more averse to model uncertainty, and considers a larger set of alternative models. In the limit $\Omega \rightarrow 1$, the agent only considers the reference model $\mathbb{P}$, corresponding to the case of no model uncertainty. Notice also that, with this form of $f(\xi)$, we can express

$$
\sup _{V} \mathbb{E}^{\mathbb{P} \xi}[f(\xi) U(V)]=\sup _{V} \mathbb{E}^{\mathbb{P}}[\xi U(V)+\Omega \xi \log \xi(-U(V))]
$$


where we use the fact that $\xi$ is a change of measure. The first term is the standard expected utility term, computed under the alternative measure $\mathbb{P}^{\xi}$. The quantity $\xi \log \xi$ is the relative entropy between the reference measure $\mathbb{P}$ and the alternative measure $\mathbb{P}^{\xi}$ and captures the distance between the two models in log-likelihood space. Unlike Hansen and Sargent [2008], we scale the relative entropy by next period's utility. ${ }^{7}$ Scaling by $U(V)$ has the dual advantages of preserving the importance of model uncertainty when wealth $V$ increases and maintaining analytical tractability. In our one period setting, this is the direct analog of scaling by the agent's value function, as in Maenhout [2004].

Finally, we can rewrite the optimization problem (9) as

$$
\begin{array}{ll}
\inf _{\xi}\left\{\operatorname { s u p } _ { V } \left\{\mathbb{E}^{\mathbb{P}}[\xi f(\xi) U(V)] \quad\right.\right. \text { such that } & \left.\mathbb{E}^{\mathbb{P}}[y V]=V_{0}\right\} . \\
& \text { such that } \left.\quad \mathbb{E}^{\mathbb{P}}[\xi]=1, \xi>0, \xi f(\xi) \geq 0\right\},
\end{array}
$$

where $y=\xi m^{\xi}$ is the pricing kernel of the uncertainty averse agent under the reference measure $\mathbb{P}$. Of the four constraints in (12), the first corresponds to the initial budget constraint, the second enforces that $\mathbb{P}^{\xi}$ is a probability measure, the third that $\xi$ is a valid change of measure, and the fourth ensures that $\xi f(\xi) U(V)$ is non-decreasing in $V$. For future use, we denote the set of admissible changes of measure (parametrizing the set of alternative models)

$$
\Xi=\left\{\xi: \mathbb{E}^{\mathbb{P}}[\xi]=1, \xi>0, \xi f(\xi) \geq 0\right\}
$$

\footnotetext{
${ }^{7}$ In the one period version of the setup of Hansen and Sargent [2008], the investor solves$$
\inf _{\xi} \sup _{V} \mathbb{E}^{\mathbb{P}}[\xi U(V)+\theta \xi \log \xi], \quad \theta \in[\bar{\theta}, \infty], \bar{\theta}>0
$$

where $\theta$ is a positive constant which controls aversion to model uncertainty. In their setup, finite values of $\theta$ generate aversion to model uncertainty while $\theta=\infty$ corresponds to forcing their (additive) penalty term $\theta \mathbb{E}^{\mathbb{P}}[\xi \log \xi]$ to be so large that $\xi$ is forced to be identically equal to one and hence is the limiting case of no aversion to model uncertainty.
} 


\subsection{The model-uncertainty-induced utility function}

We turn now to the notion of the model-uncertainty-induced utility function. Switching the $\operatorname{order}^{8}$ of min and max, we can rewrite (12) as

$$
\sup _{V}\left\{\inf _{\xi \in \Xi}\left\{\mathbb{E}^{\mathbb{P}}[\xi f(\xi) U(V)]\right\} \quad \text { such that } \quad \mathbb{E}^{\mathbb{P}}[y V]=V_{0}\right\} \text {. }
$$

In this subsection, we focus on the inner minimization $\inf _{\xi \in \Xi}\left\{\mathbb{E}^{\mathbb{P}}[\xi f(\xi) U(V)]\right\}$. Introduce

$$
\Psi \equiv \frac{1}{\Omega}-1, \quad(\text { note } \Psi \leq 0), \text { and } \quad g(\xi) \equiv \xi-\log \xi-1
$$

and let $\min _{s}\{-U(V)\}$ and $\max _{s}\{-U(V)\}$ denote the minimum and maximum values of $-U(V) \equiv-U(V(s))$ across the $S$ possible states. Before we solve for the worst-case model, we summarize some properties of $\inf _{\xi \in \Xi}\left\{\mathbb{E}^{\mathbb{P}}[\xi f(\xi) U(V)]\right\}$.

Proposition 3 (1) $\inf _{\xi \in \Xi}\left\{\mathbb{E}^{\mathbb{P}}[\xi f(\xi) U(V)]\right\} \leq \mathbb{E}^{\mathbb{P}}[U(V)]$.

(2) In the special case of $\Omega=1, \xi(s) \equiv 1$ in all states $s=1, \ldots, S$ and the inequality in the first part holds with equality.

(3) As $\Omega$ increases, $\inf _{\xi \in \Xi}\left\{\mathbb{E}^{\mathbb{P}}[\xi f(\xi) U(V)]\right\}$ is non-increasing (and strictly decreasing except in the degenerate case of $U(V)$ being independent of the state $s)$.

Thus, the expected present value of the agent's utility is at most that under the reference measure. Intuitively, since the reference measure $\mathbb{P}$ is in the set of possible alternative $\mathcal{P}$, the uncertainty averse agent cannot do better than her utility under the reference measure. The second part of the proposition shows that the case $\Omega=1$ corresponds to no aversion to model uncertainty, while the last part shows that letting $\Omega$ tend towards infinity corresponds to total aversion to model uncertainty. Hansen and Sargent [2008] show (section 7.4) that their formulation of robustness has, at high enough levels of aversion to model uncertainty,

\footnotetext{
${ }^{8}$ since the argument is concave in $V$ and convex in $\xi$
} 
a "breakdown point" at which the agent's objective is no longer concave in her wealth. Our formulation does not suffer from this complication. In fact, in the next proposition, we show that $\xi$ is bounded, implying that $\inf _{\xi \in \Xi}\left\{\mathbb{E}^{\mathbb{P}}[\xi f(\xi) U(V)]\right\}$ is bounded below.

Proposition 4 The worst-case likelihood satisfies

$$
\inf _{\xi \in \Xi}\left\{\mathbb{E}^{\mathbb{P}}[\xi f(\xi) U(V)]\right\}=\max _{\eta}\left\{\sum_{s=1}^{S} \mathbb{P}(s)[(1+\Omega g(\xi(s)))(U(V)-\beta(s))]\right\}
$$

where $\beta(s)$ are the Lagrange multipliers on the constraints $\xi(s) f(\xi(s)) \geq 0$ and

$$
\xi(s)=\exp \left(\left(1+\frac{\eta}{(U(V)-\beta(s))}\right) \Psi\right) .
$$

In the degenerate case that $\min _{s}\{-U(V)\}=\max _{s}\{-U(V)\}, \xi(s) \equiv 1$ and we set $\beta(s) \equiv 0$. Otherwise,

$$
\beta(s)= \begin{cases}0, & \text { if } U(V) \neq 0 \text { and }\left(1+\frac{\eta}{U(V)}\right) \Psi \leq \log \xi^{\text {crit }} \\ \frac{\eta \Psi}{\left(\Psi-\log \xi^{\text {crit }}\right)}+U(V), & \text { otherwise. }\end{cases}
$$

Furthermore, $\eta$ satisfies $\eta \geq 0$ and $\eta$ and $\xi(s)$ are bounded:

$$
\begin{aligned}
& \qquad \min _{s}\{-U(V)\} \leq \eta \leq \max _{s}\{-U(V)\} \\
& \text { and for all } s=1, \ldots, S: \quad \xi(s) \in\left[\exp (\Psi), \xi^{\text {crit }}\right]
\end{aligned}
$$

with $\xi^{\text {crit }}$ the unique point for which $f\left(\xi^{\text {crit }}\right)=0$, given by $\xi^{\text {crit }}=\exp (1 / \Omega)$.

Using the results of Proposition 4, we can now define the model-uncertainty-induced utility function to be

$$
U^{\mathrm{MU}}(V)=(1+\Omega g(\xi))(U(V)-\beta)
$$


where $\xi$ is the worst-case distortion given by (14) (evaluated at the maximizing value $\hat{\eta}$ of $\eta$ in (14)). The optimization in (13) thus becomes

$$
\sup _{V}\left\{\mathbb{E}^{\mathbb{P}}\left[U^{\mathrm{MU}}(V)\right] \quad \text { such that } \quad \mathbb{E}^{\mathbb{P}}[y V]=V_{0}\right\}
$$

Notice that, since the worst-case distortion $\xi$ depends both on the reference measure $\mathbb{P}$ and the space of basis assets, the model-uncertainty-induced utility function $U^{\mathrm{MU}}(V)$ is also setting-specific. The following proposition confirms that $U^{\mathrm{MU}}(V)$ is a valid utility function and establishes some of its other properties.

Proposition $5 U^{\mathrm{MU}}(V)$ satisfies Assumption 2; in particular, (i), $U^{\mathrm{MU}}(V) \leq U(V) \leq 0$. Furthermore, (ii), $U^{\mathrm{MU}^{\prime}}(V)=\xi f(\xi) U^{\prime}(V) \geq 0$, and, (iii), if $\Omega>1$ and $\min _{s}\{-U(V)\} \neq$ $\max _{s}\{-U(V)\}$, then $U^{\mathrm{MU}^{\prime}}(V)$ is less than, equal to or greater than $U^{\prime}(V)$ according to whether $U(V)$ is greater than, equal to or less than $-\hat{\eta}$, where $\hat{\eta}$ is the maximizing value of $\eta$ in (14). Finally, (iv), the coefficient of absolute risk aversion of $U^{\mathrm{MU}}(V)$ satisfies

$$
-\frac{U^{\mathrm{MU}^{\prime \prime}}(V)}{U^{\mathrm{MU}^{\prime}}(V)} \equiv \operatorname{CARA}\left(U^{\mathrm{MU}}((V)) \geq \operatorname{CARA}\left(U((V)) \equiv-\frac{U^{\prime \prime}(V)}{U^{\prime}(V)}\right.\right.
$$

with equality holding in the inequality in (21) if $\Omega=1$.

Intuitively, the uncertainty averse agent places higher probabilities (i.e. $U^{\mathrm{MU}^{\prime}}(V)>U^{\prime}(V)$ ) on lower utility outcomes. Furthermore, model uncertainty increases the effective coefficient of absolute risk aversion. Since (by a Taylor expansion), $1+\Omega g(\xi) \approx 1+\Omega(\xi-1)^{2} / 2$, the amount by which $U^{\mathrm{MU}}(V)$ is less than $U(V)$ is largest when $\xi$ is furthest away from one. This increases the curvature and, hence, the coefficient of absolute risk aversion, of the effective utility function $U^{\mathrm{MU}}$. This is a direct analog to the intuition of Barillas et al. [2009] that the optimizing decisions of an uncertainty averse agent is observationally equivalent to one with Epstein and Zin [1989] preferences. Further economic intuition on the modeluncertainty-induced utility function is that it can potentially explain the equity premium 
puzzle of Mehra and Prescott [1985]. Since this property is not essential for "No Good Deals", we have relegated our analysis of this to Appendix C.

\subsection{Restriction on the pricing kernel under model uncertainty}

We now return to considering problem (12). The certainty equivalent CE of problem (12) is the solution to

$$
\sup _{V}\left\{\inf _{\xi \in \Xi}\left\{\mathbb{E}^{\mathbb{P}}[\xi f(\xi) U(V)]\right\} \text { such that } \mathbb{E}^{\mathbb{P}}[y V]=V_{0}\right\}=\inf _{\xi \in \Xi}\left\{\mathbb{E}^{\mathbb{P}}\left[\xi f(\xi) U\left(V_{0}+\mathrm{CE}\right)\right]\right\}
$$

Using the results of Propositions 3 and 4, we can rewrite the above as

$$
\sup _{V}\left\{\mathbb{E}^{\mathbb{P}}\left[U^{\mathrm{MU}}(V)\right] \text { such that } \mathbb{E}^{\mathbb{P}}[y V]=V_{0}\right\}=U\left(V_{0}+\mathrm{CE}\right) \text {. }
$$

To solve for the certainty equivalent CE in equation (22), we use the methodology of Cox and Huang [1989] (or section 2 of Černý [2003]). We state the result for truncated quadratic utility in the following proposition - the cases of exponential and CRRA utility are in Appendix B.

Proposition 6 Let $\bar{\beta}>0$ be the investor's subjective discount factor and $\xi$ be the worst-case distortion.

For the case of truncated quadratic utility, $U(V)=-\bar{\beta}(\max (\bar{V}-V, 0))^{2}$, with bliss point $\bar{V}$ (where it is assumed that $V_{0}+\mathrm{CE}<\bar{V}$ ), the certainty equivalent is

$$
\begin{aligned}
& \mathrm{CE}=\frac{1}{\operatorname{CARA}\left(U\left(V_{0}\right)\right)}\left(1-\frac{1}{\sqrt{\mathbb{E}^{\mathbb{P}}\left[\frac{y^{2}}{\xi f(\xi)}\right]}}\right), \quad \text { and furthermore } \\
& \mathrm{CE} \leq C \Longleftrightarrow \mathbb{E}^{\mathbb{P}}\left[\frac{y^{2}}{\xi f(\xi)}\right] \leq\left(\frac{1}{1-\left(\operatorname{CARA}\left(U\left(V_{0}\right)\right) C\right)}\right)^{2} .
\end{aligned}
$$

Equation (24) is the analog to the Cochrane and Saá-Requejo [2000] bound and clearly, our bound reduces to theirs if $\xi \equiv 1$. Equations (B.2) and (B.4) in Appendix B extend 
bounds for exponential and CRRA utility functions in Černý [2003] to the presence of model uncertainty.

\section{Good Deal bounds}

In this section, we develop the good deal bounds that are implied by the pricing kernel restrictions derived in Proposition 6. We focus on the case of truncated quadratic utility, Section 4.3 and Appendix B consider the exponential and CRRA utility cases.

The setup is the same as in Section 2. Briefly, there are $N_{b}$ basis assets. The time 0 price of basis asset $i$ is $p_{i}$ and, at time 1 , it pays $x_{i} \equiv x_{i}(s)$, in state $s$, for each $i=1, \ldots, N_{b}$. The pricing kernel $y$ must reprice the basis assets exactly. There is a focus asset which pays $x_{c} \equiv x_{c}(s)$, at time 1 . We continue to make Assumption 1 .

Notice that the model-uncertainty-induced utility function $U^{\mathrm{MU}}(V)$ satisfies the technical assumptions that Černý $[2003]^{9}$ imposes to derive the relationship (in the absence of model uncertainty) between bounds on the certainty equivalent and bounds on the pricing kernel. This leads us to our definition of good deal bounds under model uncertainty - which we term No Good Deals - No Bad Models.

Definition 7 Under model uncertainty, the lower $\underline{C}$ and upper $\bar{C}$ good deal bounds on the time 0 price of the focus asset solve

$$
\begin{aligned}
& \underline{C}=\inf _{\xi \in \Xi}\left\{\inf _{y}\left\{\mathbb{E}^{\mathbb{P}}\left[y x_{c}\right] \text { such that } \mathbb{E}^{\mathbb{P}}[y \boldsymbol{x}]=\boldsymbol{p}, y \geq 0, \mathbb{E}^{\mathbb{P}}\left[\frac{y^{2}}{\xi f(\xi)}\right] \leq A^{2}\right\}\right\} \\
& \bar{C}=\sup _{\xi \in \Xi}\left\{\sup _{y}\left\{\mathbb{E}^{\mathbb{P}}\left[y x_{c}\right] \text { such that } \mathbb{E}^{\mathbb{P}}[y \boldsymbol{x}]=\boldsymbol{p}, y \geq 0, \mathbb{E}^{\mathbb{P}}\left[\frac{y^{2}}{\xi f(\xi)}\right] \leq A^{2}\right\}\right\} .
\end{aligned}
$$

The first two restrictions are the same as in the traditional good deal bounds and enforce, respectively, that the candidate pricing kernel prices the basis assets and implies absence of arbitrage opportunities. The third restriction is new and replaces the bound on the volatility

\footnotetext{
${ }^{9}$ See Černý [2003] Theorems 2 and 3.
} 
of the pricing kernel by the bound on $\mathbb{E}^{\mathbb{P}}\left[\frac{y^{2}}{\xi f(\xi)}\right]$. Notice that the infimum (respectively, supremum) over admissible distortions $\xi$ in the expression for the lower good deal bound $\underline{C}$ (upper good deal bound $\bar{C}$ ) is equivalent to taking the worst-case distortion in maximizing the expected future utility of wealth, as we show below. Notice also that, for (25) to have a solution, the bound $A^{2}$ has to be large enough to reprice the basis assets. In particular, we require that $A^{2} \geq A^{\star 2}$, with

$$
A^{\star 2}=\inf _{\xi \in \Xi}\left\{\inf _{y}\left\{\mathbb{E}^{\mathbb{P}}\left[\frac{y^{2}}{\xi f(\xi)}\right] \quad \text { such that } \mathbb{E}^{\mathbb{P}}[y \mathbf{x}]=\mathbf{p} \text { and } y \geq 0\right\}\right\} \text {. }
$$

Equation (26) ensures that the set $\Xi$ of admissible distortions is non-empty.

Similarly to Cochrane and Saá-Requejo [2000], we consider two different combinations of slack and binding constraints. In

Case (1) The constraint $\mathbb{E}^{\mathbb{P}}\left[\frac{y^{2}}{\xi f(\xi)}\right] \leq A^{2}$ binds (the constraint $y \geq 0$ may be binding or slack). The solution is stated in Proposition 8.

Case (2) The constraint $\mathbb{E}^{\mathbb{P}}\left[\frac{y^{2}}{\xi f(\xi)}\right] \leq A^{2}$ is slack. The solution is stated in Proposition 9.

To simplify the solution, we introduce a binary variable $\mathbf{1}_{L / U}$ which takes the value 1 (respectively, -1 ) if we are computing the lower (upper) good deal bound $\underline{C}$ (respectively, $\bar{C}$ ). Denoting by $\delta$ a scalar, interpretable as the Lagrange multiplier on $\mathbb{E}^{\mathbb{P}}\left[\frac{y^{2}}{\xi f(\xi)}\right] \leq A^{2}$, and by $\varphi$ a normalization constant, we define the loss function $Z\left(s, \mathbf{1}_{L / U}, \mathbf{w}, \varphi, \delta\right)$ in state $s$ as

$$
\begin{aligned}
Y\left(s, \mathbf{1}_{L / U}, \mathbf{w}, \varphi, \delta\right) & \equiv \max \left[-\mathbf{1}_{L / U} \frac{\left(\varphi x_{c}(s)-\mathbf{w}^{\prime} \mathbf{x}(s)\right)}{\delta}, 0\right] \\
Z\left(s, \mathbf{1}_{L / U}, \mathbf{w}, \varphi, \delta\right) & \equiv\left(Y\left(s, \mathbf{1}_{L / U}, \mathbf{w}, \varphi, \delta\right)\right)^{2}=-U^{T}\left(\mathbf{1}_{L / U} \frac{\left(\varphi x_{c}(s)-\mathbf{w}^{\prime} \mathbf{x}(s)\right)}{\delta}\right)
\end{aligned}
$$

where $\mathbf{w}$ is a $N_{b}$ - dimensional vector, interpretable as Lagrange multipliers on the constraint $\mathbb{E}^{\mathbb{P}}[y \mathbf{x}]=\mathbf{p}$. We denote the minimum (maximum) value of $Z\left(s, \mathbf{1}_{L / U}, \mathbf{w}, \varphi, \delta\right)$ across the $S$ possible states by $\min _{s}\left\{Z\left(s, \mathbf{1}_{L / U}, \mathbf{w}, \varphi, \delta\right)\right\}\left(\max _{s}\left\{Z\left(s, \mathbf{1}_{L / U}, \mathbf{w}, \varphi, \delta\right)\right\}\right)$. 


\subsection{Case (1): The constraint $\mathbb{E}^{\mathbb{P}}\left[\frac{y^{2}}{\xi f(\xi)}\right] \leq A^{2}$ binds}

We solve for the good deal bounds $\underline{C}$ and $\bar{C}$ by forming the Lagrangian of the constrained optimization problem.

Proposition 8 When $\mathbb{E}^{\mathbb{P}}\left[\frac{y^{2}}{\xi f(\xi)}\right] \leq A^{2}$ binds, the investor's marginal utility in state $s$ is

$$
y(s)=\xi(s) f(\xi(s)) Y\left(s, \boldsymbol{1}_{L / U}, \boldsymbol{w}, 1, \delta\right),
$$

where the worst-case distortion $\xi$ and the Lagrange multipliers $\beta(s)$ on $\xi(s) f(\xi(s)) \geq 0$ are given in Proposition 4 , with the utility of terminal wealth $U(V)$ replaced by $-Z\left(s, \mathbf{1}_{L / U}, \boldsymbol{w}, 1, \delta\right)$. The lower $\underline{C}$ and upper $\bar{C}$ good deal bounds solve

$$
\begin{aligned}
\underline{C}=\max _{\delta>0, \boldsymbol{w}}\left\{\boldsymbol{w}^{\prime} \boldsymbol{p}-\frac{1}{2} \delta A^{2}+\right. \\
\max _{\eta}\left\{\sum_{s=1}^{S} \mathbb{P}(s)\left[-\frac{1}{2} \delta(1+\Omega g(\xi(s)))\left(Z\left(s, \boldsymbol{1}_{L / U}, \boldsymbol{w}, 1, \delta\right)+\beta(s)\right)\right]\right\}, \\
\bar{C}=\min _{\delta>0, \boldsymbol{w}}\left\{\boldsymbol{w}^{\prime} \boldsymbol{p}+\frac{1}{2} \delta A^{2}+\right. \\
\left.\min _{\eta}\left\{\sum_{s=1}^{S} \mathbb{P}(s)\left[\frac{1}{2} \delta(1+\Omega g(\xi(s)))\left(Z\left(s, \mathbf{1}_{L / U}, \boldsymbol{w}, 1, \delta\right)+\beta(s)\right)\right]\right\}\right\}
\end{aligned}
$$

Equivalently, with $U^{T}(V) \equiv-(\max (-V, 0))^{2}$

$$
\begin{array}{ll}
\underline{C} \text { solves : } & A^{2}=\min _{\xi \in \Xi}\left\{\max _{\mu>0, \boldsymbol{v}}\left\{2 \boldsymbol{v}^{\prime} \boldsymbol{p}-2 \mu \underline{C}+\mathbb{E}^{\mathbb{P}}\left[\xi f(\xi) U^{T}\left(\mu x_{c}-\boldsymbol{v}^{\prime} \boldsymbol{x}\right)\right]\right\},\right. \\
\bar{C} \text { solves : } & A^{2}=\min _{\xi \in \Xi}\left\{\max _{\mu<0, \boldsymbol{v}}\left\{2 \boldsymbol{v}^{\prime} \boldsymbol{p}-2 \mu \bar{C}+\mathbb{E}^{\mathbb{P}}\left[\xi f(\xi) U^{T}\left(\mu x_{c}-\boldsymbol{v}^{\prime} \boldsymbol{x}\right)\right]\right\} .\right.
\end{array}
$$

Equations (30) and (31) are solved numerically by choice of $\eta$ and then by choice of $\delta>0$, w.

Consider first the pricing kernel, (29). The quantity $Y\left(s, \mathbf{1}_{L / U}, \mathbf{w}, 1, \delta\right)$ is the Cochrane and Saá-Requejo [2000] pricing kernel (in the absence of model uncertainty). The uncertainty 
averse investor distorts that pricing kernel, assigning greater marginal utility to states with worse outcomes. In the special case of $\Omega=1$ (which corresponds to no aversion to model uncertainty), $\xi(s)=1$ in each state, the pricing kernel (29) reduces to the pricing kernel of Cochrane and Saá-Requejo [2000] and the lower and upper good deal bounds (30) and (31) coincide with those of Cochrane and Saá-Requejo [2000]. Similarly, when the loss function $Z\left(s, \mathbf{1}_{L / U}, \mathbf{w}, 1, \delta\right)$ is independent of the state $s, \xi(s)=1$ in each state and the solution reduces once gain to the solution of Cochrane and Saá-Requejo [2000].

In the more general case of $\Omega$ strictly greater than one (so that the investor exhibits aversion to model uncertainty) and $\min _{s}\left\{Z\left(s, \mathbf{1}_{L / U}, \mathbf{w}, 1, \delta\right)\right\} \neq \max _{s}\left\{Z\left(s, \mathbf{1}_{L / U}, \mathbf{w}, 1, \delta\right)\right\}$ (so that the investor is not indifferent amongst the different states of the world), $\xi(s)$ decreases when $Z\left(s, \mathbf{1}_{L / U}, \mathbf{w}, 1, \delta\right)$ increases. Since $\xi(s) f(\xi(s))$ is decreasing in $\xi(s)$, the marginal utility $y(s)$ of the state $s$ increases as $Z\left(s, \mathbf{1}_{L / U}, \mathbf{w}, 1, \delta\right)$ increases. Thus, the uncertainty averse agent assigns higher marginal utility to states with larger losses. The maximization (respectively, minimization) over $\delta, \mathrm{w}$ for the lower good deal bound $\underline{C}$ (respectively, the upper good deal bound $\bar{C}$ ) then has the effect of minimizing the average weighted losses.

Notice that the Lagrange multipliers $\mathbf{w}$ have the interpretation of optimal hedging positions for the focus asset in the basis assets. This property allows us to interpret $Y\left(s, \mathbf{1}_{L / U}, \mathbf{w}, 1, \delta\right)$ as the loss in state $s$ after (optimally) hedging the focus asset and $Z\left(s, \mathbf{1}_{L / U}, \mathbf{w}, 1, \delta\right)$ as a (post-hedge) loss function.

\subsection{Case (2): The constraint $\mathbb{E}^{\mathbb{P}}\left[\frac{y^{2}}{\xi f(\xi)}\right] \leq A^{2}$ is slack}

When $\mathbb{E}^{\mathbb{P}}\left[\frac{y^{2}}{\xi f(\xi)}\right] \leq A^{2}$ is slack, the good deal bounds reduce to the arbitrage bounds (that is, those enforceable by sub- or super-replication). In particular, since the infimum (respectively, 
supremum) over $\xi$ in the case of the lower (respectively, upper) good deal bound becomes irrelevant, we solve

$$
\begin{aligned}
& \underline{C}=\inf _{y}\left\{\mathbb{E}^{\mathbb{P}}\left[y x_{c}\right] \text { such that } \mathbb{E}^{\mathbb{P}}[y \mathbf{x}]=\mathbf{p}, y \geq 0\right\}, \quad \text { and } \\
& \bar{C}=\sup _{y}\left\{\mathbb{E}^{\mathbb{P}}\left[y x_{c}\right] \text { such that } \mathbb{E}^{\mathbb{P}}[y \mathbf{x}]=\mathbf{p}, y \geq 0\right\}
\end{aligned}
$$

the solution of which can always be obtained numerically (see Cochrane and Saá-Requejo [2000]) since it is a linear program. We denote by $C_{\mathrm{Arb}}\left(\mathbf{1}_{L / U}\right)$ the respective arbitrage bound. The arbitrage bound $C_{\mathrm{Arb}}\left(\mathbf{1}_{L / U}\right)$ is the good deal bound if the implied pricing kernel is such that the constraint $\mathbb{E}^{\mathbb{P}}\left[\frac{y^{2}}{\xi f(\xi)}\right] \leq A^{2}$ is slack. To check, we solve

$$
\min _{\xi \in \Xi}\left\{\min _{y}\left\{\mathbb{E}^{\mathbb{P}}\left[\frac{y^{2}}{\xi f(\xi)}\right] \quad \text { such that } C_{\text {Arb }}\left(\mathbf{1}_{L / U}\right)=\mathbb{E}^{\mathbb{P}}\left[y x_{c}\right], \mathbb{E}^{\mathbb{P}}[y \mathbf{x}]=\mathbf{p} \text { and } y \geq 0\right\}\right\}
$$

If the minimized objective in (35) is less than $A^{2}$, then $C_{\mathrm{Arb}}\left(\mathbf{1}_{L / U}\right)$ is the good deal bound. Otherwise, the constraint is binding and case (1) is the relevant one. The solution is summarized below, with $\mathbf{v}$ a $N_{b}$ - dimensional vector, the Lagrange multipliers on the constraint $\mathbb{E}^{\mathbb{P}}[y \mathbf{x}]=\mathbf{p}$

Proposition 9 When $\mathbb{E}^{\mathbb{P}}\left[\frac{y^{2}}{\xi f(\xi)}\right] \leq A^{2}$ is slack, the investor's marginal utility in state $s$ is

$$
y(s)=\xi(s) f(\xi(s)) Y(s, 1, \boldsymbol{v}, \mu, 1)
$$

where the worst-case distortion $\xi$ and the Lagrange multipliers $\beta(s)$ on $\xi(s) f(\xi(s)) \geq 0$ are given in Proposition 4, with the utility of terminal wealth $U(V)$ replaced by $-Z(s, 1, \boldsymbol{v}, \mu, 1)$. The solution to (35) is

$$
\max _{\boldsymbol{v}, \mu}\left\{2 \boldsymbol{v}^{\prime} \boldsymbol{p}-2 \mu C_{\mathrm{Arb}}\left(\boldsymbol{1}_{L / U}\right)+\max _{\eta}\left\{\sum_{s=1}^{S} \mathbb{P}(s)[-(1+\Omega g(\xi(s)))(Z(s, 1, \boldsymbol{v}, \mu, 1)+\beta(s))]\right\}\right\} .
$$


Equivalently, if

$$
A^{2}>\min _{\xi \in \Xi}\left\{\max _{\boldsymbol{v}, \mu}\left\{2 \boldsymbol{v}^{\prime} \boldsymbol{p}-2 \mu C_{\mathrm{Arb}}\left(\boldsymbol{1}_{L / U}\right)+\mathbb{E}^{\mathbb{P}}\left[\xi f(\xi) U^{T}\left(\mu x_{c}-\boldsymbol{v}^{\prime} \boldsymbol{x}\right)\right]\right\}\right\}
$$

then $\underline{C}$ (or respectively, $\bar{C}$ ) equals the lower (or upper) arbitrage bound $C_{\mathrm{Arb}}\left(\boldsymbol{1}_{L / U}\right)$.

Problem (37) is solved numerically by choice of $\eta$ and then by choice of $\mathbf{v}, \mu$.

Similarly to case (1), the uncertainty averse agent assigns higher marginal utility to states with larger losses. Unlike case (1), however, the Lagrange multipliers $\mathbf{v}$ do not have the interpretation of optimal hedges for the focus asset. Instead, the optimal hedges $\mathbf{w}$ enforce the arbitrage bounds in (34) and can be computed explicitly by solving the dual to the linear program. Specifically, w solves

$$
\max _{\mathbf{w}} \mathbf{w}^{\prime} \mathbf{p} \quad \text { such that } \quad \mathbf{w}^{\prime} \mathbf{x}(s) \leq x_{c}(s) \text {, for each state } s, s=1, \ldots, S,
$$

for the lower bound (for the upper bound, replace max by min and $\leq$ by $\geq$ ).

To conclude this subsection, we reemphasize the intuition of the "No Good Deals" methodology. Interpreting $A^{2}$ as the target level of a reward-for-risk measure (a Sharpe ratio or certainty equivalent), the "No Good Deals" methodology computes lower and upper good deals bounds which either (1) achieve the target level of the reward-for-risk measure under the worst-case likelihood, or (2) are the arbitrage bounds $C_{\mathrm{Arb}}\left(\mathbf{1}_{L / U}\right)$ when the target level cannot be achieved. Furthermore, by analogy with (35) and (38), the minimum level of the restriction on the pricing kernel $A^{\star 2}$ (see equation (26)) has the dual representation

$$
A^{\star 2}=\min _{\xi \in \Xi}\left\{\max _{\mathbf{v}}\left\{2 \mathbf{v}^{\prime} \mathbf{p}+\mathbb{E}^{\mathbb{P}}\left[\xi f(\xi) U^{T}\left(-\mathbf{v}^{\prime} \mathbf{x}\right)\right]\right\}\right\} .
$$

Thus, $A^{\star 2}$ is the maximum reward-for-risk available under the worst-case measure from trading in the basis assets. The restriction $A^{2} \geq A^{\star 2}$ can then be interpreted as the requirement that the target reward-for-risk exceeds that available from trading in the basis assets alone. 


\subsection{Good deal bounds under exponential and CRRA utility}

We derive the restrictions on the pricing kernel for exponential and for CRRA utility in Appendix B. Similarly, to the dual utility function $U^{T}(V)$, define the dual utility functions $U^{E}(V) \equiv-\exp (-V-1)$ and $U^{\mathrm{CRRA}}(V) \equiv-(\max (-V, 0))^{1-\gamma} /\left(\gamma(1-1 / \gamma)^{1-\gamma}\right)$ and similarly to (27) and (28), define $Y^{E}\left(s, \mathbf{1}_{L / U}, \mathbf{w}, \varphi, \delta\right), Z^{E}\left(s, \mathbf{1}_{L / U}, \mathbf{w}, \varphi, \delta\right)$ and $Y^{\mathrm{CRRA}}\left(s, \mathbf{1}_{L / U}, \mathbf{w}, \varphi, \delta\right)$, $Z^{\mathrm{CRRA}}\left(s, \mathbf{1}_{L / U}, \mathbf{w}, \varphi, \delta\right)$ for exponential and CRRA utility, respectively, as

$$
\begin{aligned}
Y^{E}\left(s, \mathbf{1}_{L / U}, \mathbf{w}, \varphi, \delta\right) & \equiv \exp \left(\left(-\mathbf{1}_{L / U} \frac{\left(\varphi x_{c}(s)-\mathbf{w}^{\prime} \mathbf{x}(s)\right)}{\delta}\right)-1\right) \\
Z^{E}\left(s, \mathbf{1}_{L / U}, \mathbf{w}, \varphi, \delta\right) & \equiv Y^{E}\left(s, \mathbf{1}_{L / U}, \mathbf{w}, \varphi, \delta\right)=-U^{E}\left(\mathbf{1}_{L / U} \frac{\left(\varphi x_{c}(s)-\mathbf{w}^{\prime} \mathbf{x}(s)\right)}{\delta}\right), \\
Y^{\mathrm{CRRA}}\left(s, \mathbf{1}_{L / U}, \mathbf{w}, \varphi, \delta\right) & \equiv \frac{1}{(1-1 / \gamma)^{-\gamma}}\left(\max \left[-\mathbf{1}_{L / U} \frac{\left(\varphi x_{c}(s)-\mathbf{w}^{\prime} \mathbf{x}(s)\right)}{\delta}, 0\right]\right)^{-\gamma}, \\
Z^{\mathrm{CRRA}}\left(s, \mathbf{1}_{L / U}, \mathbf{w}, \varphi, \delta\right) & \equiv-U^{\mathrm{CRRA}}\left(\mathbf{1}_{L / U} \frac{\left(\varphi x_{c}(s)-\mathbf{w}^{\prime} \mathbf{x}(s)\right)}{\delta}\right) .
\end{aligned}
$$

The solution to the good deals problem under exponential and CRRA utility is similar (full details are in Appendix B) to that given in Propositions 8 and 9, except that

(1) $Y$ and $Z$ are replaced by $Y^{E}$ and $Z^{E}$ or by $Y^{\mathrm{CRRA}}$ and $Z^{\mathrm{CRRA}}$, respectively;

(2) $U^{T}$ (truncated quadratic) is replaced by $U^{E}$ (exponential) or $U^{\text {CRRA }}$ (CRRA);

(3) the factors $1 / 2$ and 2 in (30), (31), (32), (33), (37) and (38) are replaced by 1.

Notice that, the investor with exponential utility assigns greater weight to the tails of the loss distribution compared to the truncated quadratic utility case. In particular, while for the latter, the loss function $Z$ computes the squared deviation from zero profit, for the former, the loss function $Z^{E}$ computes the exponential deviation. Thus, the loss functions inherit the structure of the utility function used to derive the restriction on the pricing kernel. In particular, as we noted in Propositions 8 and 9 , the negative of the loss functions $Z, Z^{E}$ and $Z^{\mathrm{CRRA}}$ take the role of the utility of terminal wealth $U(V)$ in computing the good deal bounds. Just as $U(V)$ is concave, so $Z, Z^{E}$ and $Z^{\text {CRRA }}$ are convex. 
This convexity property enables us to show that the impact of model uncertainty on the good deal bounds depends on the dispersion in the loss function $Z$ across the $S$ possible states of the world. To make the argument more concrete, define

$$
F\left(Z^{\mathrm{adj}}\right) \equiv(1+\Omega g(\xi)) Z^{\mathrm{adj}}, \quad \text { where } Z^{\mathrm{adj}} \equiv Z+\beta
$$

Notice that $Z$ can refer to either $Z\left(s, \mathbf{1}_{L / U}, \mathbf{w}, 1, \delta\right)$ or $Z(s, 1, \mathbf{v}, \mu, 1)$ (or $Z^{E}$ or $Z^{\mathrm{CRRA}}$ ) and that $g(\xi)$ depends on $Z^{\text {adj }}$ through $\xi$. It is straightforward (the calculations are similar to the proof of Proposition 5) to verify that $F^{\prime \prime}\left(Z^{\text {adj }}\right) \geq 0$ and $F^{\prime \prime \prime}\left(Z^{\text {adj }}\right) \leq 0$. By a Taylor expansion with exact remainder:

$$
\begin{aligned}
\mathbb{E}^{\mathbb{P}}\left[F\left(Z^{\text {adj }}\right)\right]= & \mathbb{E}^{\mathbb{P}}[Z]+\frac{1}{2} \sum_{s=1}^{S} \mathbb{P}(s)\left[\left(Z^{\text {adj }}-\mathbb{E}^{\mathbb{P}}\left[Z^{\text {adj }}\right]\right)^{2} F^{\prime \prime}\left(Z^{\text {adj } \star}\right), \quad\right. \text { or } \\
\mathbb{E}^{\mathbb{P}}\left[F\left(Z^{\text {adj }}\right)\right]=\mathbb{E}^{\mathbb{P}}[Z]+ & \frac{1}{2} \mathbb{V a r} r^{\mathbb{P}}\left[Z^{\text {adj }}\right] F^{\prime \prime}\left(\mathbb{E}^{\mathbb{P}}\left[Z^{\text {adj }}\right]\right) \\
& +\frac{1}{6} \sum_{s=1}^{S} \mathbb{P}(s)\left[\left(Z^{\text {adj }}-\mathbb{E}^{\mathbb{P}}\left[Z^{\text {adj }}\right]\right)^{3} F^{\prime \prime \prime}\left(Z^{\text {adj } \star \star}\right),\right.
\end{aligned}
$$

for some $Z^{\text {adj } \star}$ and $Z^{\text {adj } \star \star}$. Now comparing with (30), (31) and (37), we see the sense in which a wide dispersion in $Z$ increases, in magnitude, the difference between good deal bounds in the presence of model uncertainty and in its absence. In the latter case, good deal bounds depend only upon $\mathbb{E}^{\mathbb{P}}\left[Z\left(s, \mathbf{1}_{L / U}, \mathbf{w}, 1, \delta\right)\right]$ (or $\mathbb{E}^{\mathbb{P}}[Z(s, 1, \mathbf{v}, \mu, 1)]$ in determining if $\mathbb{E}^{\mathbb{P}}\left[\frac{y^{2}}{\xi f(\xi)}\right] \leq A^{2}$ is slack), but in the presence of model uncertainty, they depend upon higher moments. Ceteris paribus, the larger the variance of (and/or the more negatively skewed) $Z\left(s, \mathbf{1}_{L / U}, \mathbf{w}, 1, \delta\right)$, the wider the good deal bounds become. Similar comments apply in making lower, the solution to problem (37). When $\mathbb{E}^{\mathbb{P}}\left[\frac{y^{2}}{\xi f(\xi)}\right] \leq A^{2}$ is slack (case (2)), the good deal bounds can widen out no more and are equal to the arbitrage bounds $C_{\mathrm{Arb}}\left(\mathbf{1}_{L / U}\right)$. 


\subsection{General properties of the good deal bounds}

In this subsection, we develop some general properties of the good deal bounds. All these properties are equally applicable to good deal bounds constructed from restrictions on the pricing kernel in $(24),(B .2)$ or $(B .4)$.

Proposition 10 (a) Good deal bounds in the presence of model uncertainty are never narrower than those in its absence.

(b) As either $A^{2}$ increases or $\Omega$ increases, the lower good deal bound $\underline{C}$ is non-increasing and the upper good deal bound $\bar{C}$ is non-decreasing.

Property (a) is intuitive: Since the investor can always choose the reference model $\mathbb{P}$ as the worst-case model, the no good deals - no bad models good deal bounds must be at least as wide as the traditional good deal bounds (i.e. in the absence of model uncertainty). Similarly, as the restriction on the pricing kernel is relaxed ( $A^{2}$ increases) or the investor becomes more averse to model uncertainty ( $\Omega$ increases), the good deal bounds widen (until they reach the arbitrage bounds).

For the following, let $\mathbf{u}$ denote an arbitrary $N_{b}$ - dimensional vector.

Proposition 11 Let $\lambda$ be a positive constant. If the lower and upper good deal bounds for a focus payoff $x_{c}$ are $\underline{C}$ and $\bar{C}$, respectively, with corresponding optimal hedges $\underline{\mathbf{w}}$ and $\overline{\mathbf{w}}$, then the lower and upper good deal bounds for a focus payoff $\lambda x_{c}+\mathbf{u}^{\prime} \mathbf{x}$ are $\lambda \underline{C}+\mathbf{u}^{\prime} \mathbf{p}$ and $\lambda \bar{C}+\mathbf{u}^{\prime} \mathbf{p}$, respectively, with corresponding optimal hedges $\lambda \underline{\mathbf{w}}+\mathbf{u}$ and $\lambda \overline{\mathbf{w}}+\mathbf{u}$.

Corollary 12 Consider a focus payoff of the form $\boldsymbol{u}^{\prime} \boldsymbol{x}$. Then the lower and upper good deal bounds coincide and are both equal to $\boldsymbol{u}^{\prime} \boldsymbol{p}$, and the optimal hedges are $\boldsymbol{u}$.

Thus, the good deal bounds satisfy linearity with respect to adding portfolios of basis assets and homogeneity with respect to positive multiples of the focus asset payoff. Furthermore, as shown in Corollary 12, redundant assets are priced exactly at their replication cost. Proposition 13 shows that the good deal bounds satisfy also a dominance property. 
Proposition 13 Consider two focus assets, $A$ and $B$, with payoffs $x_{c}^{A}$ and $x_{c}^{B}$, respectively, with corresponding lower and upper good deal bounds $\underline{C}^{A}, \bar{C}^{A}, \underline{C}^{B}$ and $\bar{C}^{B}$. If $x_{c}^{A}(s) \leq x_{c}^{B}(s)$ for each state $s$, and the good deal bounds are computed using the same basis assets, then

$$
\underline{C}^{A} \leq \underline{C}^{B} \quad \text { and } \quad \bar{C}^{A} \leq \bar{C}^{B}
$$

The last three results are direct counterparts of the properties (see Hodges [1998]) of good deal bounds in the absence of model uncertainty.

\section{$5 \quad$ Estimating the $\Omega$ parameter and the choice of $A^{2}$}

In this section, we describe a procedure for estimating the degree of uncertainty aversion, $\Omega$, and discuss the choice of $A^{2}$. The methodology for estimating $\Omega$ is based on the error detection probability methodology of Hansen and Sargent [2008], Anderson et al. [2003] and Maenhout [2004]. In particular, we choose $\Omega$ so that the maximum reward-for-risk achievable under the worst-case likelihood is statistically indistinguishable from the maximum rewardfor-risk under the reference model. While some investors or financial institutions may exhibit more uncertainty aversion than others, in this paper we follow the (conservative) recommendations of Hansen and Sargent [2008], Anderson et al. [2003] and Maenhout [2004] and choose $\Omega$ such that the error detection probability is between $20 \%$ and $10 \%$. $\Omega$ is computed from a historical data-set of asset prices, making the parameter $\Omega$ context-specific, with different data-sets or different assets leading to different estimates of $\Omega$.

Consider $A^{\star 2}$ in equations (26) and (40). $A^{\star 2}$ is the maximum reward-for-risk available from trading in the basis assets under the worst-case model and solves

$$
A^{\star 2}(\Omega) \equiv A^{\star 2}=\max _{\mathbf{v}}\left\{2 \mathbf{v}^{\prime} \mathbf{p}+\max _{\alpha}\left\{\sum_{s=1}^{S} \mathbb{P}(s)[-(1+\Omega g(\xi(s)))(Z(s, 1, \mathbf{v}, 0,1)+\beta(s))]\right\}\right\}
$$


where $\beta(s)$ and $\xi(s)$ are defined as in problem (37) but replacing $Z(s, 1, \mathbf{v}, \mu, 1)$ by $Z(s, 1, \mathbf{v}, 0,1)$. Given an estimate of $\Omega$, we can solve (45) numerically for $A^{\star 2}$. To estimate $\Omega$, we search numerically for the value of $\Omega$ which makes the solution to problem (45) statistically difficult to distinguish (at the chosen error detection probability) from the solution to the equivalent problem setting $\xi(s) \equiv 1$

$$
A^{\star \mathrm{NoMU} 2} \equiv \max _{\mathbf{v}}\left\{2 \mathbf{v}^{\prime} \mathbf{p}+\sum_{s=1}^{S} \mathbb{P}(s)[-(Z(s, 1, \mathbf{v}, 0,1))]\right\}
$$

We solve problems (45) and (46) using historical data, setting the number of states $S$ equal to the number of available observations $J$ and assigning equal probabilities to each state $\mathbb{P}(s)=1 / J$. We bootstrap the historical data, $K$ times, by repeatedly sampling from the data with replacement. Sampling with replacement means that sometimes we sample a given historical data point more than once and sometimes not at all. For each bootstrapped sample, we compute the solution to problem (46). We sort the $K$ solutions into order and select the solution corresponding to the chosen error detection probability. We then set $\Omega$ to be that value which gives the same solution to problem (45) for the full (non-bootstrapped) historical sample. Note that when solving problems (45) and (46), we work with returns (i.e. we divide each historical observed price by its price at the preceding time point). Hence, $Z(s, 1, \mathbf{v}, 0,1)$ now reflects "payoffs in return form". 10

We now turn our attention to choosing the bound $A^{2}$ in the constraint $\mathbb{E}^{\mathbb{P}}\left[\frac{y^{2}}{\xi f(\xi)}\right] \leq A^{2}$ : There are different alternatives:

\footnotetext{
${ }^{10}$ As an alternative, we could work with excess (i.e. over and above the risk-free rate) returns which may be better conditioned. If we denote the excess returns by $\mathbf{R}$, then problem (45) can be rewritten:

$$
\min _{\xi \in \Xi}\left\{\min _{y}\left\{\mathbb{E}^{\mathbb{P}}\left[\frac{y^{2}}{\xi f(\xi)}\right] \quad \text { such that } \mathbb{E}^{\mathbb{P}}[y \mathbf{R}]=\mathbf{0}, y \geq 0 \text { and } \mathbb{E}^{\mathbb{P}}[y]=1 /\left(1+R^{f}\right)\right\}\right\},
$$

where $R^{f}$ is the average one period risk-free rate. We need the extra condition $\mathbb{E}^{\mathbb{P}}[y]=1 /\left(1+R^{f}\right)$ to normalise $y$ - without it, the minimizing value of $y$ is zero. The problem is easily solved by analogy to problems (45) and (46).
} 
(1) Choose $A^{2}$ to be some margin over (or some multiple $\geq 1$ of) either (a) $A^{\star N o M U} 2$ or (b) $A^{\star 2}$ (with the former being more conservative).

(2) Choose $A^{2}$ through the choice of an annualized Sharpe ratio $h_{\mathrm{Ann}}$.

Expanding upon the latter, Černý [2003] shows that the certainty equivalent CE of any utility function $U^{G}(V)$ and its coefficient of absolute risk aversion $\operatorname{CARA}\left(U^{G}\left(V_{0}\right)\right)$ are linked, under Assumptions 1 and 2, to investment opportunities, over a time period $\Delta t$, with a small per period Sharpe ratio $h_{\text {PerP }}$ by:

$$
\operatorname{CARA}\left(U^{G}\left(V_{0}\right)\right) \mathrm{CE} \approx \frac{1}{2} h_{\mathrm{PerP}}^{2}=\frac{1}{2} h_{\mathrm{Ann}}^{2} \Delta t
$$

Hence, a bound on $\mathrm{CE} \leq C$ in equation (24) is approximately the same as a bound on $\mathbb{E}^{\mathbb{P}}\left[\frac{y^{2}}{\xi f(\xi)}\right] \leq\left(\frac{1}{1-\left(\operatorname{CARA}\left(U\left(V_{0}\right)\right) C\right)}\right)^{2} \approx\left(\frac{1}{1-\frac{1}{2} h_{\mathrm{PerP}}^{2}}\right)^{2} \approx 1+h_{\mathrm{PerP}}^{2}=1+h_{\mathrm{Ann}}^{2} \Delta t$, by a Taylor expansion. This relates the bound on $\mathbb{E}^{\mathbb{P}}\left[\frac{y^{2}}{\xi f(\xi)}\right]$ to a bound on the annualised Sharpe ratio

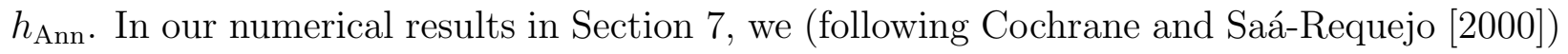
set $A^{2}=\left(1+h_{\mathrm{Ann}}^{2} \Delta t\right) /(1+r)^{2}$, for a time period $\Delta t$ years, where $r$ is the per period risk-free interest-rate.

In choosing $h_{\text {Ann }}$, we note that Hansen and Sargent [2008], Anderson et al. [2003] and Maenhout [2004] find that, for very plausible error detection probabilities, model uncertainty can account for half or more of the excess return on equity markets. ${ }^{11}$ The presence of model uncertainty can thus reduce the maximal admissible Sharpe ratio $h_{\text {Ann }}$ of Cochrane and SaáRequejo [2000]. Instead, we interpret $h_{\mathrm{Ann}}$ as the maximal Sharpe ratio achievable under

\footnotetext{
${ }^{11}$ The impact of model uncertainty on asset returns has also been studied by Epstein and Wang [1994], Chen and Epstein [2002], Uppal and Wang [2003], Cao et al. [2005], Barillas et al. [2009] and Anderson et al. [2009].
} 
the worst-case measure. In our numerical results, we will choose ${ }^{12}$ various values of $h_{\mathrm{Ann}}$ between $1 / 3$ and 0.5 .

\section{$6 \quad$ Multiple time periods}

The previous analysis considered a one period problem. In this section, we extend the results to a multi-period setting, focusing on the two period case. There are three dates, indexed by $t=0,1,2$. At time $t=0$, the investor takes a position in the focus asset, which pays $x_{c}$ at time $t=2$. Time $t=1$ is an intermediate rebalancing time, when the investor can adjust her hedging portfolio in the basis assets and update her valuation of the focus asset. Denote the time $t=0,1$ conditional expectation under the reference measure $\mathbb{P}$ by $\mathbb{E}_{t}^{\mathbb{P}}$, and the time $t=1,2$ pricing kernel and worst-case distortion by $y_{t}$ and $\xi_{t}$, respectively. Finally, denote the time $t=0,1$ lower and upper good deal bounds by $\underline{C}_{t}$ and $\bar{C}_{t}$, respectively. With this notation, the two period problem (for the lower bound) is

$$
\begin{gathered}
\underline{C}_{0}=\inf _{\xi_{1}, \xi_{2}}\left\{\operatorname { i n f } _ { y _ { 1 } , y _ { 2 } } \left\{\mathbb{E}_{0}^{\mathbb{P}}\left[y_{1} y_{2} x_{c}\right] \text { such that } \mathbb{E}_{0}^{\mathbb{P}}\left[y_{1} \mathbf{p}_{1}\right]=\mathbf{p}_{0}, y_{1} \geq 0, \mathbb{E}_{0}^{\mathbb{P}}\left[\frac{y_{1}^{2}}{\xi_{1} f\left(\xi_{1}\right)}\right] \leq A_{0}^{2},\right.\right. \\
\left.\mathbb{E}_{1}^{\mathbb{P}}\left[y_{2} \mathbf{x}\right]=\mathbf{p}_{1} \quad \forall \Im_{1}, y_{2} \geq 0, \mathbb{E}_{1}^{\mathbb{P}}\left[\frac{y_{2}^{2}}{\xi_{2} f\left(\xi_{2}\right)}\right] \leq A_{1}^{2} \quad \forall \Im_{1}\right\} \\
\text { such that } \left.\mathbb{E}_{0}^{\mathbb{P}}\left[\xi_{1}\right]=1, \xi_{1}>0, \xi_{1} f\left(\xi_{1}\right) \geq 0, \mathbb{E}_{1}^{\mathbb{P}}\left[\xi_{2}\right]=1, \xi_{2}>0, \xi_{2} f\left(\xi_{2}\right) \geq 0\right\}
\end{gathered}
$$

where $\Im_{1}$ is the information set at time $t=1$. Thus, in a two period setting, the initial lower good deal bound imposes sequential constraints on the pricing kernels at each date.

\footnotetext{
${ }^{12}$ In 2000, the then chairman of Barclays bank said he wanted its investment banking arm to achieve a $20 \%$ target return on equity (when risk-free interest-rates were around $5 \%$ and the volatility of Barclays shares was around 25\%) - which could be interpreted as implying he wanted it to achieve an ex-post Sharpe ratio of $(20-5) / 25=0.6$. Choosing $h_{\mathrm{Ann}}=0.6$ is then equivalent to seeking to achieve his target, even under the worst-case likelihood.
} 
Applying the Law of Iterated Expectations (see the detailed proof in Cochrane and SaáRequejo [2000]), we can rewrite equation (49) as two (sequential) one period problems

$$
\begin{gathered}
\underline{C}_{1}\left(s_{1}\right)=\inf _{\xi_{2}}\left\{\inf _{y_{2}}\left\{\mathbb{E}_{1}^{\mathbb{P}}\left[y_{2} x_{c}\right] \text { such that } \mathbb{E}_{1}^{\mathbb{P}}\left[y_{2} \mathbf{x}_{2}\right]=\mathbf{p}_{1}, y_{2} \geq 0, \mathbb{E}_{1}^{\mathbb{P}}\left[\frac{y_{2}^{2}}{\xi_{2} f\left(\xi_{2}\right)}\right] \leq A_{1}^{2}\right\},\right. \\
\text { such that } \left.\mathbb{E}_{1}^{\mathbb{P}}\left[\xi_{2}\right]=1, \xi_{2}>0, \xi_{2} f\left(\xi_{2}\right) \geq 0\right\} \\
\underline{C}_{0}=\inf _{\xi_{1}}\left\{\inf _{y_{1}}\left\{\mathbb{E}_{0}^{\mathbb{P}}\left[y_{1} \underline{C}_{1}\right] \text { such that } \mathbb{E}_{0}^{\mathbb{P}}\left[y_{1} \mathbf{x}_{1}\right]=\mathbf{p}_{0}, y_{1} \geq 0, \mathbb{E}_{0}^{\mathbb{P}}\left[\frac{y_{1}^{2}}{\xi_{1} f\left(\xi_{1}\right)}\right] \leq A_{0}^{2}\right\},\right. \\
\text { such that } \left.\mathbb{E}_{0}^{\mathbb{P}}\left[\xi_{1}\right]=1, \xi_{1}>0, \xi_{1} f\left(\xi_{1}\right) \geq 0\right\},
\end{gathered}
$$

where equation (50) takes the solution to the time 1 problem as given. For the upper bound $\bar{C}_{0}$, we solve equation (49) or (50) with the infimum operator replaced by the supremum operator. Notice that this separation of the non-sequential problem (49) into two sequential problems is possible because the constraints on the pricing kernels and worst-case distortions are applied sequentially and the pricing kernels preclude arbitrage opportunities. As pointed out in Hansen and Sargent [2008] and Maccheroni, Marinacci, and Rustichini [2006], the multiplier problem (11) enforces commitment on the part of the uncertainty averse agent, making it possible to express the restrictions on the pricing kernel recursively. Finally, notice that, with this formulation, we can easily extend the no good deals methodology to accommodate more than two periods.

Consider now solving for the optimal hedging portfolios that achieve the good deal bounds at each date. Denote by $\mathbf{w}_{t}$ the optimal hedging portfolio at time $t=0,1$. Then, similarly to the good deal bounds, the hedging strategy solves the sequential ${ }^{13}$ problems

\footnotetext{
${ }^{13}$ For illustration, we implicitly assume that the constraints $\mathbb{E}_{0}^{\mathbb{P}}\left[\frac{y_{1}^{2}}{\xi_{1} f\left(\xi_{1}\right)}\right] \leq A_{0}^{2}$ and $\mathbb{E}_{1}^{\mathbb{P}}\left[\frac{y_{2}^{2}}{\xi_{2} f\left(\xi_{2}\right)}\right] \leq A_{1}^{2}$ bind.
} 


$$
\begin{aligned}
\underline{C}_{1}\left(s_{1}\right) & =\max _{\delta_{1}\left(s_{1}\right)>0, \mathbf{w}_{1}\left(s_{1}\right)}\left\{\mathbf{w}_{1}^{\prime}\left(s_{1}\right) \mathbf{p}_{1}\left(s_{1}\right)-\frac{1}{2} \delta_{1}\left(s_{1}\right) A_{1}^{2}\right. \\
& \left.+\max _{\eta_{1}}\left\{\sum_{s_{2}=1}^{S} \mathbb{P}\left(s_{2}\right)\left[-\frac{1}{2} \delta_{1}\left(s_{1}\right)\left(1+\Omega g\left(\xi_{2}\left(s_{2}\right)\right)\right)\left(Z\left(s_{2}, \mathbf{1}_{L / U}, \mathbf{w}_{1}, 1, \delta_{1}\left(s_{1}\right)\right)+\beta_{1}\left(s_{2}\right)\right)\right]\right\}\right\} \\
\underline{C}_{0} & =\max _{\delta_{0}>0, \mathbf{w}_{0}}\left\{\mathbf{w}_{0}^{\prime} \mathbf{p}_{0}-\frac{1}{2} \delta_{0} A_{0}^{2}\right. \\
& \left.+\max _{\eta_{0}}\left\{\sum_{s_{1}=1}^{S} \mathbb{P}\left(s_{1}\right)\left[-\frac{1}{2} \delta_{0}\left(1+\Omega g\left(\xi_{1}\left(s_{1}\right)\right)\right)\left(Z\left(s_{1}, \mathbf{1}_{L / U}, \mathbf{w}_{0}, 1, \delta_{0}\right)+\beta_{0}\left(s_{1}\right)\right)\right]\right\}\right\}
\end{aligned}
$$

Let $\hat{\eta}_{1}$ be the optimal value of $\eta_{1}$ and $\hat{\delta}_{1}\left(s_{1}\right)$ the optimal value of $\delta_{1}\left(s_{1}\right)$ in $(51)$. Using the first-order conditions, problem (51) reduces to

$$
\begin{aligned}
\underline{C}_{1}\left(s_{1}\right) & =\max _{\mathbf{w}_{1}\left(s_{1}\right)}\left\{\mathbf{w}_{1}^{\prime}\left(s_{1}\right) \mathbf{p}_{1}\left(s_{1}\right)-\hat{\delta}_{1}\left(s_{1}\right) \underline{A}_{1}^{2}\right\}, \quad \text { where } \\
\underline{A}_{1}^{2} & =A_{1}^{2}+\sum_{s_{2}=1, \xi_{2}=\xi^{\text {crit }}}^{S}\left[\frac{1}{2}\left(1-\xi^{\text {crit }}\right) \Omega \hat{\eta}_{1} \Psi\right] .
\end{aligned}
$$

Then

$$
\begin{aligned}
\underline{C}_{0} & =\max _{\delta_{0}>0, \mathbf{w}_{0}}\left\{\mathbf{w}_{0}^{\prime} \mathbf{p}_{0}-\frac{1}{2} \delta A_{0}^{2}\right. \\
& \left.+\max _{\eta_{0}}\left\{\sum_{s_{1}=1}^{S} \mathbb{P}\left(s_{1}\right)\left[-\frac{1}{2} \delta_{0}\left(1+\Omega g\left(\xi_{1}\left(s_{1}\right)\right)\right)\left(\left(\frac{\max \left[\hat{R}_{1}\left(s_{1}\right), 0\right]}{\delta_{0}}\right)^{2}+\beta_{0}\left(s_{1}\right)\right)\right]\right\}\right\},
\end{aligned}
$$

where

$$
\hat{R}_{1}\left(s_{1}\right) \equiv \mathbf{w}_{0}^{\prime} \mathbf{p}_{1}\left(s_{1}\right)-\underline{C}_{1}\left(s_{1}\right)=-\min _{\mathbf{w}_{1}\left(s_{1}\right)}\left\{\left(\mathbf{w}_{1}^{\prime}\left(s_{1}\right)-\mathbf{w}_{0}^{\prime}\right) \mathbf{p}_{1}\left(s_{1}\right)-\hat{\delta}_{1}\left(s_{1}\right) \underline{A}_{1}^{2}\right\}
$$

Using the intuition of Section 4.3, model uncertainty has a larger impact on the time 0 good deal bounds if there is more dispersion in the values of $\max \left[\hat{R}_{1}\left(s_{1}\right), 0\right]$. Assume 
(for intuition purposes) that $\hat{\delta}_{1}\left(s_{1}\right) \underline{A}_{1}^{2}$ is relatively insensitive ${ }^{14}$ to the realization of the state $s_{1}$. Then greater dispersion in $\max \left[\hat{R}_{1}\left(s_{1}\right), 0\right]$ corresponds to greater dispersion in $\left(\mathbf{w}_{1}^{\prime}\left(s_{1}\right)-\mathbf{w}_{0}^{\prime}\right) \mathbf{p}_{1}\left(s_{1}\right)$. Conversely, the impact of model uncertainty is reduced when $\left(\mathbf{w}_{1}^{\prime}\left(s_{1}\right)-\mathbf{w}_{0}^{\prime}\right) \mathbf{p}_{1}\left(s_{1}\right)$ becomes less sensitive to the realization of the state $s_{1}$ and, in particular, the impact of model uncertainty on the time 0 good deal bounds is smallest when $\mathbf{w}_{0}=\mathbf{w}_{1}\left(s_{1}\right)$ for all states $s_{1}=1, \ldots, S$. The latter corresponds to a static hedging strategy, ${ }^{15}$ which keeps the hedging portfolio constant across time and shock realizations.

More generally, compare two strategies for hedging the position in a focus asset:

(1) a dynamic hedging strategy in the basis assets, with rebalancing allowed at the intermediate time $t=1$

(2) a static hedging strategy in the basis assets, with the positions chosen at time $t=0$ maintained at time $t=1$.

Clearly, an investor following the first strategy can always choose to maintain the position in the hedging portfolio at time $t=1$, so dynamic hedging is always weakly better than the static hedging strategy. Thus, the good deal bounds under the dynamic hedging strategy are never wider (even in the absence of model uncertainty) than the bounds under the static hedging strategy. As the investor becomes more uncertainty averse, however, the good deal bounds widen under both strategies and approach the arbitrage bounds. Thus, the distance (and, hence, the benefit to dynamic hedging) between the good deal bounds under dynamic and static hedging decreases as the investor becomes more uncertainty averse.

\footnotetext{
${ }^{14}$ If $\mathbb{E}_{1}^{\mathbb{P}}\left[\frac{y_{2}^{2}}{\xi_{2} f\left(\xi_{2}\right)}\right] \leq A_{1}^{2}$ is slack in state $s_{1}$, then from $(39), \underline{C}_{1}\left(s_{1}\right)$ is of the form $\mathbf{w}_{1}^{\prime}\left(s_{1}\right) \mathbf{p}_{1}\left(s_{1}\right)$ which is effectively, in the context of (52), equivalent to setting $\hat{\delta}_{1}\left(s_{1}\right) \underline{A}_{1}^{2}$ to zero.

${ }^{15}$ Carr et al. [1998] is a comprehensive reference on static hedging strategies with a number of examples.
} 


\section{Numerical examples}

In this section, we consider two numerical examples of computing the good deal bounds and the associated hedging portfolios. The first example values Arrow-Debreu securities in a three state, one period model with a risk-free bond and a defaultable bond as the basis assets. The second values options on a non-traded asset.

\subsection{Defaultable bond}

Consider an economy with two basis assets, a defaultable bond and a risk-free bond. At time 1, there are three possible states of the world, labelled "good", "poor" and "armageddon". The defaultable bond has a time 0 price of 1 and a payoff at time 1 equal to $1.2,0.6$ and 0 in states "good", "poor" and "armageddon" respectively. The "poor" and "armageddon" states are states in which the issuer of the defaultable bond defaults and the holder of the bond receives either $60 \%$ (partial recovery) or $0 \%$ (zero recovery) of the time 0 price. The risk-free bond has a time 0 price of 1 and a payoff at time 1 equal to 1 in all three states.

To illustrate our theoretical analysis, we simulate a data-set with 2000 data points. The defaultable bond pays 1.2, 0.6 and 0 on 1700, 200 and 100 dates in the sample, respectively. Hence, the probability, under $\mathbb{P}$, of states "good", "poor" and "armageddon" occurring are set at $0.85,0.1$ and 0.05 respectively. Given the simulated historical data-set, we use the bootstrap procedure of Section 5 to estimate $\Omega$. We bootstrap the data-set 65000 times, and, for the $80 \%$ confidence level, we find $\Omega=1.51$. In Table 1 , we report also the estimate of $\Omega$ for simulated data-sets with 500, 1000, 4000 and 8000, keeping the same probabilities of the three states. We see that as the length of the simulated data-set increases, the estimate of $\Omega$ increases, implying greater model uncertainty.

We consider three Arrow-Debreu securities as the focus assets. In particular, ArrowDebreu security $F_{j}$, for $j \in\{$ good, poor, armageddon $\}$ pays one dollar if state $j$ is realized.

Notice that $F_{\text {armageddon }}$ is a catastrophe insurance contract. It is straightforward to verify 
(or by solving (34)) that the lower and upper arbitrage bounds for the three focus assets are $2 / 3,5 / 6\left(F_{\text {good }}\right), 0,1 / 3\left(F_{\text {poor }}\right)$ and $0,1 / 6\left(F_{\text {armageddon }}\right)$ respectively. We set the maximum Sharpe ratio bound $h_{\text {Ann }}$ equal to $1 / 3$. In Table 2 , we report the lower and upper good deal bounds, $\underline{C}$ and $\bar{C}$, for different values of $\Omega$. Note that $\Omega=1$ is the no model uncertainty case. As $\Omega$ increases, the good deal bounds widen and the values of $\underline{C}$ and $\bar{C}$ get closer to the respective lower and upper arbitrage bounds and, for $\Omega=8$, the good deal bounds equal the arbitrage bounds.

Consider now the catastrophe insurance contract, $F_{\text {armageddon, }}$ in detail. In Table 3 , we report the lower $\underline{C}$ and upper $\bar{C}$ good deal bounds for different levels of uncertainty aversion, as well as the optimal hedging positions in the two basis assets. The pricing kernel constraint is slack when $\Omega=8$ for the lower bound and when $\Omega \in\{4,8\}$ for the upper bound. For these values of $\Omega$, the good deal bounds correspond to the arbitrage bounds. The positions in the defaultable bond and risk-free bond respectively (computed from equation (39)) which enforce the lower and upper arbitrage bounds are 0,0 and $-5 / 6,1 .^{16}$ In Table $3, w_{1}$ and $w_{2}$ are the optimal hedges for the defaultable bond and risk-free bond respectively. We see that, as the value of $\Omega$ changes, the optimal hedges $w_{1}$ and $w_{2}$ change significantly.

In Table 4, we focus just on the lower good deal bound and we consider a wider range of values of $\Omega$ in order to see where the boundary lies between when the constraint $\mathbb{E}^{\mathbb{P}}\left[\frac{y^{2}}{\xi f(\xi)}\right] \leq$ $A^{2}$ binds and when it is slack. We see that the boundary lies at a value of $\Omega$ of approximately 5.463. Values of $\Omega$ less than or equal to 5.4625 give a lower good deal bound where the constraint binds and values of $\Omega$ greater than or equal to 5.46375 give a lower good deal bound where the constraint is slack and hence the good deal bound is the arbitrage bound. Notice that, since the nature of the good deal bounds is discontinuous, the optimal hedges are discontinuous across the critical value of $\Omega=5.46375$.

\footnotetext{
${ }^{16}$ That is, the upper arbitrage bound $1 / 6$ can be enforced by sellng $5 / 6$ units of the defaultable bond and buying one unit of the risk-free bond which costs, at time 0 , a price of $1-5 / 6=1 / 6$ dollar while the lower arbitrage bound is enforced by zero position in each basis asset.
} 


\subsection{Options on non-traded asset}

We now consider options written on a non-traded asset. This example is similar to one in Cochrane and Saá-Requejo [2000] (p111-113) except, of course, we also consider the impact of model uncertainty. There is a traded asset, labelled 1 whose price is denoted by $S$, and a non-traded asset, labelled 2 whose value is denoted by $V$. The option (focus asset) payoff is $\max (V-K, 0)$ for a call (respectively, $\max (K-V, 0)$ for a put), where $K$ is a fixed strike. We consider a European call with $K=65$, a European call with $K=55$ and a European put with $K=65$. The option maturity is 2 years. The traded asset 1 is is correlated (but not perfectly) with the non-traded asset and can be used to partially hedge the option.

We model the underlying dynamics as double trinomial (a pyramid rather than a triangle) with steps one month apart (24 steps over 2 years). At each time-step $i(i=0,1, \ldots, 23)$ of the trinomial tree, when the price of the traded asset 1 is $S_{i}$, the price can stay the same, go up to $S_{i} \exp \left(\lambda_{1} \sigma_{1} \sqrt{d t}\right)$ or down to $S_{i} \exp \left(-\lambda_{1} \sigma_{1} \sqrt{d t}\right)$ where $\lambda_{1}=\sqrt{(3 / 2)}, \sigma_{1}=0.25$ (the volatility). and $d t=2 / 24$ (one month) is the time period corresponding to each step. The correlation between log-changes in $S$ and $V$ is $\rho$ (we set $\rho=0.8$ ). When the value of the non-traded asset 2 is $V_{i}$, the value can change to: $V_{i} \exp \left(\lambda_{2} \sigma_{2} \sqrt{d t}\left(\sqrt{1-\rho^{2}} Z_{2}+\rho Z_{1}\right)\right)$ for $Z_{1}=-1,0,1$ and $Z_{2}=-1,0,1$ and where $\lambda_{2}=\sqrt{(3 / 2)}, \sigma_{2}=0.28$ (the volatility). This is a standard double trinomial tree construction. In the limit of small time-steps, this tree construction will approximate $S$ and $V$ being jointly log-normal (but we will not be interested in this small time-step limit - we regard the discrete-time dynamics above as specifying the actual dynamics, rather than of being an approximation to some continuous-time dynamics).

The risk-free rate is 0.03 (expressed as a continuously-compounded rate). Traded asset 1 has an initial price of asset 20 and pays a dividend yield of $2 \%$ (expressed as a continuous yield proportional to its price) or 0.02 . We include a non-zero dividend yield to illustrate that it is straightforward to do so - if asset 1 were a commodity, we could treat this as its convenience yield. In the latter case, the non-traded asset 2 could be the value of something 
which gets embedded in a "real option" (new mine, new oil field or oil refinery project) which we cannot trade but where we can (partially) hedge with an imperfectly correlated financial asset. The initial value of non-traded asset 2 is 60 .

We can trade in the basis assets (asset 1 and a one period risk-free bond) and rebalance our hedges at the start of steps $0,3,6,9,12,15,18,21$ (not every step) i.e. we rebalance every three months. There are two sources of incompleteness: The discrete-time hedging and the fact that we can only hedge with a partially correlated traded asset. For illustration, we suppose the excess return on asset 1 is 0.03 and that on the non-traded asset 2 is 0.04 . We then compute the probabilities in the double trinomial tree by requiring that they sum to one and by matching each of the first two moments of $S$ and $V$.

We use a maximum Sharpe ratio bound $h_{\mathrm{Ann}}$ equal to 0.5. We consider different values of the $\Omega$ parameter $(16,8,4,2,1.75,1.5,1.25$ and 1$)$. The results are in Table 5 , where we also report the risk-neutral price (meaning with the excess returns set to zero but computed with the same placements of tree nodes) and the Black and Scholes [1973] price (labelled BS) just for illustration - neither of these prices has a real financial meaning here since one cannot even trade the non-traded asset 2 (but the difference between the former and the latter would give an idea of the discretization error if there were to be an interest in approximating joint geometric Brownian motion by the double trinomial tree).

The lower and upper good deal bounds when there is no model uncertainty (labelled No MU and computed by setting $\xi \equiv 1$ ) are, of course, the same as when $\Omega=1$ - in line with our theoretical analysis. We observe that as $\Omega$ is increased, the good deal bounds widen - in fact, in this example, changing $\Omega$ has a huge impact. For the call options, this may be partly due to the fact that the lower and upper arbitrage bounds are very wide in this example namely, zero and infinity.

We now extend our analysis by considering American options which additionally give the holder of the option the right to exercise at the same frequency as the hedge-rebalancing i.e. at the start of steps $0,3,6,9,12,15,18,21$. We consider only the lower good deal bounds 
$\underline{C}$ (for simplicity, since these correspond to reservation prices for long positions - the upper good deal bounds $\bar{C}$ (corresponding to reservation prices for short positions) are dependent upon the exercise strategy of the option buyer). We assume that the American option is exercised when the immediate exercise value exceeds the continuation value of the lower good deal bound. The results are in Table 5 where we again report the risk-neutral price. Since the non-traded asset 2 does not pay dividends, the risk-neutral prices of the American call options are the same as their European counterparts. Defining the early exercise premium to be the value of the relevant American option minus that of its European counterpart, we see that the early exercise premia of the lower good deal bounds are substantial - even for call options - and increase with increasing values of $\Omega$. Indeed, for values of $\Omega \in\{4,8,16\}$, it is optimal to immediately exercise the options (call, $K=55$ and put, $K=65$ ) which are initially in-the-money.

It is intuitive that larger values of $\Omega$ (corresponding to greater model uncertainty) lead to larger early exercise premia since, when the option is exercised, model uncertainty becomes irrelevant. This incentivizes the uncertainty averse investor to exercise the option earlier.

Our results are significant in the context of real options since real options are often American in nature.

\section{Summary and conclusions}

In this paper, we have described a new and practical approach to dealing with model uncertainty in pricing securities or contingent claims. It is based on combining the "No Good Deals" methodology of Cochrane and Saá-Requejo [2000] with elements of the robustness framework of Hansen and Sargent [2008] and of Maenhout [2004]. In an important steppingstone, we have introduced the notion of a model-uncertainty-induced utility function and shown how model uncertainty increases an investor's effective risk aversion. We have shown how the impact of model uncertainty is to give greater weight (i.e. greater than the investor's 
marginal utility) to states in which losses are relatively large. We have shown how static hedging i.e. hedging an option by taking a static ("buy-and-hold") position in other options (as opposed to dynamic hedging with the underlying asset) may become a relatively attractive hedging strategy in the presence of model uncertainty. Uppal and Wang [2003] consider the impact of multiple sources of model uncertainty on portfolio selection. As a possible future extension of our work, it would be interesting to consider the impact of multiple sources of model uncertainty (for example, different degrees of uncertainty in the estimates of the drifts or volatilities of two different assets). 


\section{References}

Evan Anderson, Lars Peter Hansen, and Thomas J. Sargent. A quartet of semigroups for model specification, robustness, prices of risk and model detection. Journal of the European Economic Association, 1(1):68-123, 2003.

Evan Anderson, Eric Ghysels, and Jennifer Juergens. The impact of risk and uncertainty on expected returns. Journal of Financial Economics, 94:233-263, 2009.

Francisco Barillas, Lars Peter Hansen, and Thomas J. Sargent. Doubts or variability? Journal of Economic Theory, 144(6):2388-2418, 2009.

Basel III. Basel III: A global regulatory framework for more resilient banks and banking systems. Basel Committee on Banking Supervision, December 2010.

A. Bernardo and O. Ledoit. Gain, loss and asset pricing. Journal of Political Economy, 108: 144-172, 2000.

Fisher Black and Myron Scholes. The pricing of options and corporate liabilities. Journal of Political Economy, 81:637-654, 1973.

P. Bossaerts, P. Ghirardato, S. Guarnaschelli, and W. Zame. Ambiguity in asset markets: Theory and experiment. Review of Financial Studies, 23:1325-1359, 2010.

Nina Boyarchenko. Ambiguity Shifts and the 2007-2008 Financial Crisis. Journal of Monetary Economics, 59(5):493-507, 2012.

P. Boyle, S. Feng, W. Tian, and T. Wang. Robust Stochastic Discount Factors. Review of Financial Studies, 21(3):1077-1122, 2008.

P. Boyle, L. Garlappi, R. Uppal, and T. Wang. Keynes meets Markowitz: The trade-off between familiarity and diversification. Management Science, 58:253-272, 2012.

Marco Cagetti, Lars Peter Hansen, Thomas J. Sargent, and Noah Williams. Robustness and pricing with uncertain growth. Review of Financial Studies, 15:363-404, 2002.

John Y. Campbell. Consumption-based asset pricing. In G.M. Constantinides, M. Harris and R. Stulz, editors, Handbook of the Economics of Finance, 2003. Elsevier Science, 2003.

H. H. Cao, T. Wang, and H. H. Zhang. Model uncertainty, limited participation and asset prices. Review of Financial Studies, 18:1219-1251, 2005.

Peter Carr, K. Ellis, and V. Gupta. Static hedging of exotic options. Journal of Finance, 53 (3):1165-1190, 1998.

Ales Černý. Generalised Sharpe ratios and asset pricing in incomplete markets. European Finance Review, 7:191-233, 2003. 
Ales Černý and Stewart Hodges. The theory of good-deal pricing in financial markets. In H. Geman, D. Madan, S. R. Pliska, and T. Vorst, editors, Mathematical Finance: Selected proceedings of the First Bachelier Congress, Paris, 2000. Springer-Verlag, 2001.

Zengjing Chen and Larry G. Epstein. Ambiguity, risk and asset returns in continuous time. Econometrica, 70:1403-1443, 2002.

John Cochrane. Asset Pricing. Princeton University Press, 2005.

John Cochrane and Jesus Saá-Requejo. Beyond arbitrage: "Good-Deal" pricing asset price bounds in incomplete markets. Journal of Political Economy, 108:79-119, 2000.

I. Cooper and E. Kaplanis. Home bias in equity portfolios, inflation hedging and international capital market equilibrium. Review of Financial Studies, 7:45-60, 1994.

Joshua Coval and Tobias Moskowitz. Home bias at home: Local equity preference in domestic portfolios. Journal of Finance, 54:2045-2073, 1999.

John Cox and Chi-fu Huang. Optimal Consumption and Portfolio Policies when Asset Prices follow a Diffusion Process. Journal of Economic Theory, 49:33-83, 1989.

J. Cvitanic, A. Lazrak, L. Martellini, and F. Zapatero. Dynamic Portfolio Choice with Parameter Uncertainty and the Economic Value of Analysts' Recommendations. Review of Financial Studies, 19:1113-1156, 2011.

Itamar Drechsler. Uncertainty, Time-Varying Fear and Asset Prices. Journal of Finance, 2012.

Daniel Ellsberg. Risk, Ambiguity and the Savage Axiom. Quarterly Journal of Economics, 75:643-669, 1961.

Larry G. Epstein and Tan Wang. Intertemporal asset pricing under Knightian uncertainty. Econometrica, 62(3):283-322, 1994.

Larry G. Epstein and Stanley E. Zin. Substitution, risk aversion and the temporal behavior of consumption and asset returns: A theoretical framework. Econometrica, 57:937-969, 1989.

L. Garlappi, R. Uppal, and T. Wang. Portfolio Selection with Parameter and Model Uncertainty: A Multi-Prior Approach. Review of Financial Studies, 20:41-81, 2007.

Itzhak Gilboa and David Schmeidler. Maxmin expected utility with non-unique priors. Journal of Mathematical Economics, 18:141-153, 1989.

Lars Peter Hansen and Ravi Jagannathan. Implications of security market data for models of dynamic economies. Journal of Political Economy, 99:225-262, 1991.

Lars Peter Hansen and Thomas J. Sargent. Robust control and model uncertainty. American Economic Review, 91:60-66, 2001. 
Lars Peter Hansen and Thomas J. Sargent. Robustness. Princeton University Press, 2008.

Lars Peter Hansen and Thomas J. Sargent. Discounted linear exponential quadratic gaussian control. IEEE Trans. Autom. Control, 40:968-971, 1995.

Lars Peter Hansen, Thomas J. Sargent, and Thomas D. Tallarini, Jr. Robust permanent income and pricing. Review of Economic Studies, 66:873-907, 1999.

Stewart Hodges. Good deal bounds. In Rama Cont, editor, Encyclopedia of Quantitative Finance. Wiley, 2009.

Stewart Hodges. A generalization of the Sharpe ratio and its application to valuation bounds and risk measures. Working paper, University of Warwick Business School, United Kingdom, 1998.

G. Huberman. Familiarity breeds investment. Review of Financial Studies, 14:659-680, 2001.

Markus Leippold, Fabio Trojani, and Paolo Vanini. Learning and asset prices under ambiguous information. Review of Financial Studies, 21:2565-2597, 2008.

Jun Liu, Jun Pan, and Tan Wang. An equilibrium model of rare-event premia and its implication for option smirks. Review of Financial Studies, 18:131-164, 2005.

F. Maccheroni, M. Marinacci, and A. Rustichini. Ambiguity aversion, roubustness and the variational representation of preferences. Econometrica, 74(6):1447-1498, 2006.

Pascal J. Maenhout. Robust portfolio rules and asset pricing. Review of Financial Studies, 17:951-983, 2004.

Rajnish Mehra and Edward C. Prescott. The equity premium: A puzzle. Journal of Monetary Economics, 15(2):145-162, 1985.

Stephen Ross. The arbitrage theory of capital asset pricing. Journal of Economic Theory, 13:341-360, 1976.

Raman Uppal and Tan Wang. Model misspecification and under-diversification. Journal of Finance, LIX:2465-2486, 2003. 


\section{Appendix A: Proofs of propositions}

\section{Proof of Proposition 3.}

(1) follows from Jensen's inequality applied to $g(\xi)$, (2) follows since $\xi(s) \equiv 1$ in all states $s$ if $\Omega=1$. For (3), note that, for any $b_{2} \geq b_{1}$ and any $a$, $\exp \left(a b_{2}\right)-\left(a b_{2}\right)-1 \geq \exp \left(a b_{1}\right)-$ $\left(a b_{1}\right)-1$, with equality only if $a=0$ or $b_{2}=b_{1}$. Applying this result with $b=\Psi$ and $a=1+\eta /(U(V)-\beta(s))$ gives part (3).

\section{Proof of Proposition 4.}

Introducing Lagrange multipliers $\alpha, \beta(s) \geq 0$ and $\varpi(s) \geq 0$, for each $s$, on the set of constraints $\Xi$, the inner minimization in problem (13) becomes:

$$
\begin{aligned}
\inf _{\xi \in \Xi}\left\{\mathbb{E}^{\mathbb{P}}[\xi f(\xi) U(V)]\right\}=\max _{\varpi(s) \geq 0, \beta(s) \geq 0, \alpha}\left\{\operatorname { m i n } _ { \xi ( s ) } \left\{\sum_{s=1}^{S} \mathbb{P}(s)[\xi(s) f(\xi(s)) U(V)+\alpha(\xi(s)-1)\right.\right. \\
-\beta(s) \xi(s) f(\xi(s))-\varpi(s) \xi(s)]\}\} .
\end{aligned}
$$

The first-order condition from taking partial derivatives with respect to $\xi(s)$ in each state $s$ implies:

$$
\alpha-\varpi(s)=(\beta(s)-U(V)) \Omega(\Psi-\log \xi(s)) .
$$

Equation (A.2) enables us to solve for $\xi(s)$. If $U(V)$ is the same in every state $s$ and/or $\Psi=0$, then the only solution is $\alpha=0, \varpi(s)=\beta(s)=0, \xi(s)=1$. So now let's consider opposing cases. We conjecture that the constraint $\xi(s)>0$ is not binding which means we could set $\varpi(s)=0$ for all $s$ (we will check this later). We need $\beta(s) \geq 0$ and $\xi(s) f(\xi(s)) \geq 0$ for all $s$. If $f(\xi(s))>0$, then $\beta(s)=0$ and we can solve for $\xi(s)$ from $(A .2)$, whereas if $f(\xi(s))=0$ (which means $\xi(s)=\xi^{\text {crit }}$ ), then $\beta(s)>0$ but this implies that $\beta(s)$ must be such that $(A .2)$ holds. This enables us to solve for $\xi(s)$ and $\beta(s)$ as in the statement of the proposition, in terms of which

$$
\eta \equiv \frac{\alpha}{\Omega \Psi}=\frac{\alpha}{(1-\Omega)} \quad \text { for } \Psi<0
$$

(If $\Psi=0$, the maximization over $\eta$ is irrelevant). With these values of $\xi(s)$, the condition $\xi(s)>0$ is automatically satisfied and hence $\varpi(s) \equiv 0$ as conjectured.

Substituting from $(A .2)$ implies that $(A .1)$ can be re-written in the form of (14). Equation (14), with $\xi(s)$ and $\beta(s)$ substituted, can be solved numerically by choice of $\eta$. To see what values of $\eta$ are possible, note that since $\Psi \leq 0$, for $\xi(s) \geq 1,(\Psi-\log \xi(s))$ cannot be strictly positive (and must be strictly negative if $\Psi<0$ ). Since $\mathbb{E}^{\mathbb{P}}[\xi]=\sum_{s=1}^{S} \mathbb{P}(s) \xi(s)=1, \xi(s)$ must be greater than or equal to 1 in at least one of the $S$ possible states. Hence, $\alpha$ must be less than or equal to zero (this is implied by the expression for $\xi(s)$ if $1 \leq \xi(s)<\xi^{\text {crit }}$ and by the expression for $\beta(s)$ if $\left.\xi(s)=\xi^{\text {crit }}\right)$. Hence, $\eta \geq 0$. Actually, we can strengthen this result. Since $\xi(s)$ must be less than or equal to one in at least one state, $\left(1+\frac{\eta}{U(V)}\right) \Psi$ must be less than or equal to zero in at least one state. Hence, $\eta$ must be less than or equal to $-U(V)$ for at least one state $s$ - giving an upper bound. A similar argument gives a lower 
bound and hence (18). The requirement that $\alpha \leq 0, \eta \geq 0$ implies (from (A.2)) that $\xi(s)$ is bounded below by $\exp (\Psi)$.

\section{Proof of Proposition 5.}

(i) follows since, by Jensen's inequality, $1+\Omega g(\xi) \geq 1$, for all $\xi$. (ii) Differentiating $U^{\mathrm{MU}}(V)$ :

$$
U^{\mathrm{MU}^{\prime}}(V)=\left(\Omega(1-\xi) \frac{\hat{\eta} \Psi}{(U(V)-\beta)}+(1+\Omega g(\xi))\right) U^{\prime}(V)=\xi f(\xi) U^{\prime}(V),
$$

after simplification (using $(A .2)$ ). Hence $U^{\mathrm{MU}^{\prime}}(V) \geq 0$ since $U^{\prime}(V) \geq 0$. (iii) When $U(V)<$ $-\hat{\eta}, \xi<1$ (from (15)), hence $\xi f(\xi)>1$ (from (10)). For (iv), differentiating $(A .4)$ :

$$
U^{\mathrm{MU}^{\prime \prime}}(V)=\xi f(\xi) U^{\prime \prime}(V)+\left(U^{\prime}(V)\right)^{2} \Omega \Psi^{2} \frac{\hat{\eta}^{2}}{(U(V)-\beta)^{3}} \xi .
$$

Then note $\beta \geq 0$ and (by Assumption 2) $U(V) \leq 0$.

Proof of Proposition 6.

For simplicity, within this proof, we interpret $V_{0}$ as forward initial wealth and normalise $\mathbb{E}^{\mathbb{P}}[y]=1$ - but nothing depends on this normalization. $\xi$ is the worst-case distortion given by (14) i.e. as in Proposition 4 evaluated at $\hat{\eta}$, the maximizing value of $\eta$.

We introduce a Lagrange multiplier $\lambda \equiv \lambda(s)$ in state $s$, for each $s=1, \ldots, S$ and reexpress (22) in the form: $\sup _{V}\left\{\mathbb{E}^{\mathbb{P}}\left[U^{\mathrm{MU}}(V)-\lambda\left(y V-V_{0}\right)\right]\right\}$. Differentiating with respect to $V$ and using the first part of Proposition 5 gives the first order condition: $2 \bar{\beta}(\bar{V}-V) \xi f(\xi)=\lambda y$ and the constraint $\mathbb{E}^{\mathbb{P}}[y V]=V_{0}$ then implies: $V_{0}=\mathbb{E}^{\mathbb{P}}[y] \bar{V}-\frac{\lambda}{\bar{\beta}} \mathbb{E}^{\mathbb{P}}\left[\frac{y^{2}}{2 \xi f(\xi)}\right]$. Hence, solving for $\lambda$ : $\lambda=\left(2 \bar{\beta}\left(\bar{V}-V_{0}\right)\right) / \mathbb{E}^{\mathbb{P}}\left[\frac{y^{2}}{\xi f(\xi)}\right]$. Subsituting for $V, \sup _{V}\left\{\mathbb{E}^{\mathbb{P}}\left[U^{\mathrm{MU}}(V)\right]\right\}$, subject to the constraint $\mathbb{E}^{\mathbb{P}}[y V]=V_{0}$, is:

$$
\sup _{V}\left\{\mathbb{E}^{\mathbb{P}}\left[U^{\mathrm{MU}}(V)\right]\right\}=\mathbb{E}^{\mathbb{P}}\left[-\xi f(\xi) \bar{\beta} \frac{1}{4} \frac{\lambda^{2}}{\bar{\beta}^{2}} \frac{y^{2}}{(\xi f(\xi))^{2}}\right]=-\bar{\beta} \frac{\left(\bar{V}-V_{0}\right)^{2}}{\mathbb{E}^{\mathbb{P}}\left[\frac{y^{2}}{\xi f(\xi)}\right]} .
$$

Now we solve for the certainty equivalent CE. From (22),

$$
-\bar{\beta}\left(\bar{V}-\left(V_{0}+\mathrm{CE}\right)\right)^{2}=-\bar{\beta} \frac{\left(\bar{V}-V_{0}\right)^{2}}{\mathbb{E}^{\mathbb{P}}\left[\frac{y^{2}}{\xi f(\xi)}\right]} .
$$

Taking the square root (we need the positive root) gives (23).

\section{Proof of Proposition 8.}

We focus on the lower good deal bound $\underline{C}$ ( $\bar{C}$ is similar and omitted $)$. Introducing Lagrange 
multipliers $\delta>0$, w (a $N_{b}$ - dimensional vector), $\alpha, \beta(s) \geq 0$ and $\varpi(s) \geq 0$, for each $s$, we can re-express (25) as:

$$
\begin{aligned}
\underline{C}=\max _{\varpi(s) \geq 0, \beta(s) \geq 0, \alpha}\{ & \min _{\xi(s)}\left\{\operatorname { m a x } _ { \delta > 0 , \mathbf { w } } \left\{\operatorname { m i n } _ { y ( s ) \geq 0 } \left\{\sum _ { s = 1 } ^ { S } \mathbb { P } ( s ) \left[y(s) x_{c}(s)-\mathbf{w}^{\prime}(y(s) \mathbf{x}(s)-\mathbf{p})\right.\right.\right.\right. \\
& \left.\left.\left.+\frac{1}{2} \delta\left(\frac{y(s)^{2}}{\xi(s) f(\xi(s))}-A^{2}\right)\right]\right\}\right\} \\
& \left.\left.+\sum_{s=1}^{S} \mathbb{P}(s)\left[\frac{1}{2} \alpha(\xi(s)-1)-\frac{1}{2} \delta \beta(s) \xi(s) f(\xi(s))-\frac{1}{2} \varpi(s) \xi(s)\right]\right\}\right\} .
\end{aligned}
$$

The first-order condition obtained by taking partial derivatives with respect to $y(s)$ in each state $s$ and, if necessary, enforcing the condition $y(s) \geq 0$ (we say if necessary because this constraint may or may not bind) implies (29) (we have anticipated the corresponding form for the case of the upper good deal bound and introduced $\mathbf{1}_{L / U}$ accordingly). Substituting for $y(s)$ and interchanging the orders of max and min which is justified since the minimand over $\xi(s)$ is convex and the maximands are concave, we get:

$$
\begin{aligned}
\underline{C}=\max _{\delta>0, \mathbf{w}}\left\{\operatorname { m a x } _ { \varpi ( s ) \geq 0 , \beta ( s ) \geq 0 , \alpha } \left\{\operatorname { m i n } _ { \xi ( s ) } \left\{\mathbf{w}^{\prime} \mathbf{p}-\frac{1}{2} \delta A^{2}\right.\right.\right. \\
+\sum_{s=1}^{S} \mathbb{P}(s)\left[-\frac{1}{2} \delta \xi(s) f(\xi(s)) Z\left(s, \mathbf{1}_{L / U}, \mathbf{w}, 1, \delta\right)+\frac{1}{2} \alpha(\xi(s)-1)\right. \\
\left.\left.\left.\left.\quad-\frac{1}{2} \delta \beta(s) \xi(s) f(\xi(s))-\frac{1}{2} \varpi(s) \xi(s)\right]\right\}\right\}\right\} .
\end{aligned}
$$

The first-order condition from taking partial derivatives with respect to $\xi(s)$ in each state $s$ implies:

$$
\alpha-\varpi(s)=\delta\left(Z\left(s, \mathbf{1}_{L / U}, \mathbf{w}, 1, \delta\right)+\beta(s)\right) \Omega(\Psi-\log \xi(s)) .
$$

The rest of the proof is similar to that of Proposition 4 - in fact, the solution can almost be read off (identifying $Z\left(s, \mathbf{1}_{L / U}, \mathbf{w}, 1, \delta\right)$ here with $-U(V)$ there). As in Proposition 4, $\varpi(s)=0$ and we identify $\eta \equiv \alpha /(\delta \Omega \Psi)$.

Equation (32) (similarly, (33)) is derived by taking a step back to the first part of $(A .9)$, removing the Lagrange multipliers $\alpha, \beta(s)$ and $\varpi(s)$ and then rearranging $(\mathrm{cf}(3))$. 


\section{Proof of Proposition 9.}

Introducing Lagrange multipliers $\mathbf{v}$ (a $N_{b}$ - dimensional vector), $\mu, \alpha, \beta(s) \geq 0$ and $\varpi(s) \geq 0$, for each $s$, problem (35) is equivalent to:

$$
\begin{aligned}
\max _{\varpi(s) \geq 0, \beta(s) \geq 0, \alpha}\left\{\operatorname { m i n } _ { \xi ( s ) } \left\{\operatorname { m a x } _ { \mathbf { v } , \mu } \left\{\operatorname { m i n } _ { y ( s ) \geq 0 } \left\{\sum _ { s = 1 } ^ { S } \mathbb { P } ( s ) \left[\frac{y(s)^{2}}{\xi(s) f(\xi(s))}+2 \mu\left(y(s) x_{c}(s)-C_{\mathrm{Arb}}\left(\mathbf{1}_{L / U}\right)\right)\right.\right.\right.\right.\right. \\
\left.\left.\left.\left.\left.-2 \mathbf{v}^{\prime}(y \mathbf{x}(s)-\mathbf{p})\right]\right\}\right\}+\sum_{s=1}^{S} \mathbb{P}(s)[\alpha(\xi(s)-1)-\beta(s) \xi(s) f(\xi(s))-\varpi(s) \xi(s)]\right\}\right\} .
\end{aligned}
$$

The rest of the proof is similar to that of Proposition 4 (identifying $Z(s, 1, \mathbf{v}, \mu, 1)$ here with $-U(V)$ there) and Proposition 8. Again, $\varpi(s)=0$ and we identify $\eta \equiv \alpha /(\Omega \Psi)$.

\section{Proof of Proposition 10.}

Part (a) follows because (by Jensen's inequality) $1+\Omega g(\xi(s))=1+\Omega(\xi(s)-\log \xi(s)-1) \geq 1$ for all $\xi(s)$. Part (b): The statement concerning $A^{2}$ is obvious from (25) while that concerning $\Omega$ is proven in the same way as part (3) of Proposition 3.

Proofs of Propositions 11, 12 and 13.

These are easily proven by substituting into (30), (31) and (37).

\section{Appendix B: Exponential and CRRA utility functions}

In this appendix, we detail the analysis of good deal bounds for the cases of exponential and CRRA utility functions (as opposed to the (truncated) quadratic utility function case which we have hitherto focussed on).

The extension of Proposition 6 is stated in the following proposition.

Proposition B.1 (1) For the case of exponential utility, $U(V)=-\bar{\beta} \exp (-B V)$, with constant absolute risk aversion $B>0$, the certainty equivalent is

$$
\begin{gathered}
\mathrm{CE}=\frac{1}{\operatorname{CARA}\left(U\left(V_{0}\right)\right)} \mathbb{E}^{\mathbb{P}}\left[y \log \left(\frac{y}{\xi f(\xi)}\right)\right], \\
\mathrm{CE} \leq C \Longleftrightarrow \mathbb{E}^{\mathbb{P}}\left[y \log \left(\frac{y}{\xi f(\xi)}\right)\right] \leq \operatorname{CARA}\left(U\left(V_{0}\right)\right) C .
\end{gathered}
$$

(2) For the case of constant relative risk aversion (CRRA) utility with CRRA coefficient $\gamma>1, U(V)=\bar{\beta} \frac{V^{1-\gamma}}{1-\gamma}$, the certainty equivalent is

$$
\begin{aligned}
\mathrm{CE} & =V_{0}\left(\mathbb{E}^{\mathbb{P}}\left[y\left(\frac{y}{\xi f(\xi)}\right)^{\frac{-1}{\gamma}}\right]\right)^{\frac{\gamma}{1-\gamma}}-V_{0}, \quad \text { and furthermore } \\
\mathrm{CE} \leq C & \Longleftrightarrow \mathbb{E}^{\mathbb{P}}\left[y\left(\frac{y}{\xi f(\xi)}\right)^{\frac{-1}{\gamma}}\right] \leq\left(1+\mathrm{CARA}\left(U\left(V_{0}\right)\right) \frac{C}{\gamma}\right)^{\frac{1}{\gamma}-1} .
\end{aligned}
$$


In deriving the associated good deal bounds, we replace the constraint $\mathbb{E}^{\mathbb{P}}\left[\frac{y^{2}}{\xi f(\xi)}\right] \leq A^{2}$ by (for exponential) $\mathbb{E}^{\mathbb{P}}\left[y \log \left(\frac{y}{\xi f(\xi)}\right)\right] \leq A^{2}$ or by (for CRRA) $\mathbb{E}^{\mathbb{P}}\left[y\left(\frac{y}{\xi f(\xi)}\right)^{\frac{-1}{\gamma}}\right] \leq A^{2}$.

Propositions 8 and 9, and more specifically equations (30), (31), (32), (33), (37), (38), (40), (45) and (46), hold in their entirety provided: $Y$ and $Z$ are replaced by $Y^{E}$ and $Z^{E}$ (for exponential) or by $Y^{\mathrm{CRRA}}$ and $Z^{\mathrm{CRRA}}$ (for CRRA), respectively; the dual utility function $U^{T}$ is replaced by $U^{E}(V)$ or $U^{\mathrm{CRRA}}(V)$; and the factors $1 / 2$ and 2 are replaced by 1 .

So as examples, for exponential utility, the analogs of (30) and (46), respectively, read

$$
\begin{aligned}
& \underline{C}= \max _{\delta>0, \mathbf{w}}\left\{\mathbf{w}^{\prime} \mathbf{p}-\delta A^{2}+\right. \\
& \max _{\eta}\left\{\sum_{s=1}^{S} \mathbb{P}(s)\left[-\delta(1+\Omega g(\xi(s)))\left(Z^{E}\left(s, \mathbf{1}_{L / U}, \mathbf{w}, 1, \delta\right)+\beta(s)\right)\right]\right\}, \\
& A^{\star \mathrm{NoMU} 2} \equiv \max _{\mathbf{v}}\left\{\mathbf{v}^{\prime} \mathbf{p}+\sum_{s=1}^{S} \mathbb{P}(s)\left[-\left(Z^{E}(s, 1, \mathbf{v}, 0,1)\right)\right]\right\} .
\end{aligned}
$$

The latter means that the estimate of $\Omega$ and the choice of $A^{2}$ also depend upon the choice of utility function. In choosing $A^{2}$, the same alternatives are available but there is a particularly simply way for the exponential utility case via the choice of an annualized exponential (Hodges [1998]) Sharpe ratio $h_{\mathrm{Ann}}^{E}$ because then equation (48) holds exactly, for an arbitrary time period $\Delta t$ years - indeed Hodges [1998] takes that to be the definition of $h_{\mathrm{Ann}}^{E}$. Hence, we can set $A^{2}=h_{\mathrm{Ann}}^{E 2} \Delta t$. Furthermore, for normally distributed returns and in the limit of small time periods, Hodges [1998] and Cerný [2003] show that the exponential Sharpe ratio $h_{\mathrm{Ann}}^{E}$ and the (standard) Sharpe ratio $h_{\mathrm{Ann}}$ coincide.

Finally, we stress that the arbitrage bounds in (34) and both the statements and the proofs of Propositions 11, 12 and 13 are unaffected by the choice of utility function.

\section{Appendix C: Equity premium puzzle}

In this appendix, we show that our model uncertainty framework, whilst differing from those of Hansen and Sargent [2008], Barillas et al. [2009] and Maenhout [2004], is like theirs, potentially able to explain the equity premium puzzle of Mehra and Prescott [1985].

We consider two one period economies - in the first economy, the representative agent has no model uncertainty (quantities are denoted with a superscript ${ }^{\text {NoMU }}$ ) and has CRRA utility $U(c)=\bar{\beta} \frac{c^{1-\gamma}}{1-\gamma}$ and, in the second economy, the representative agent seeks robustness against model uncertainty but is otherwise identical. Mean consumption growth is denoted by $\mu_{c}$ and the volatility of consumption growth is denoted by $\sigma_{c}$. Consumption, at time 0 , is denoted $c_{0}$. Consumption $c_{1}$ at time 1 can take on one of three values: $c_{0} \exp \left(\mu_{c} d t+\lambda \sigma_{c} \sqrt{d t}\right)$, $c_{0} \exp \left(\mu_{c} d t\right)$ or $c_{0} \exp \left(\mu_{c} d t-\lambda \sigma_{c} \sqrt{d t}\right)$, where $\lambda=\sqrt{(3 / 2)}$ and $d t=1$ (i.e. we consider a time period of one year). The one period risk-free returns are denoted by $R_{f}^{\mathrm{NoMU}}$ and $R_{f}$. There is a stock, with volatility $\sigma_{S}$, whose price is assumed to be perfectly correlated with 
Table C.1: Excess returns and risk-free rates for different values of $\gamma$ and $\Omega$

\begin{tabular}{rrrrrrrr}
\hline$\gamma$ & $\Omega$ & $\sigma(m)$ & $\sigma(\xi f(\xi) m)$ & $R_{f}^{\text {NoMU }}-1$ & $R_{f}-1$ & $E R^{\text {NoMU }}$ & $E R$ \\
\hline 2 & 2 & 6.21 & 7.76 & 3.52 & 3.44 & 1.33 & 1.66 \\
2 & 8 & 6.21 & 25.21 & 3.52 & 2.55 & 1.33 & 5.36 \\
2 & 16 & 6.21 & 49.82 & 3.52 & 1.31 & 1.33 & 10.47 \\
5 & 2 & 14.83 & 20.68 & 7.79 & 7.14 & 3.30 & 4.58 \\
5 & 8 & 14.83 & 81.83 & 7.79 & 0.48 & 3.30 & 17.02 \\
\hline
\end{tabular}

consumption. Its time 0 price is normalised to 1 and at time 1 it can take on one of three values: $\exp \left(\mu_{c} d t+\lambda \sigma_{c} \sqrt{d t}\right)^{\left(\sigma_{S} / \sigma_{c}\right)}, \exp \left(\mu_{c} d t\right)^{\left(\sigma_{S} / \sigma_{c}\right)}$ or $\exp \left(\mu_{c} d t-\lambda \sigma_{c} \sqrt{d t}\right)^{\left(\sigma_{S} / \sigma_{c}\right)}$. Standard results (Cochrane [2005]) imply that, for the first economy

$$
E R^{\mathrm{NoMU}} \equiv \mathbb{E}^{\mathbb{P}}\left[R^{\mathrm{NoMU}}\right]-R_{f}^{\mathrm{NoMU}}=-R_{f}^{\mathrm{NoMU}} \operatorname{Cov}(m, R) \quad \text { and } \quad R_{f}^{\mathrm{NoMU}}=\frac{1}{\mathbb{E}^{\mathbb{P}}[m]},
$$

where $m \equiv \bar{\beta} \frac{U^{\prime}\left(c_{1}\right)}{U^{\prime}\left(c_{0}\right)}=\bar{\beta}\left(\frac{c_{1}}{c_{0}}\right)^{-\gamma}$ denotes the pricing kernel. For the second economy,

$$
E R \equiv \mathbb{E}^{\mathbb{P}}[R]-R_{f}=-R_{f} \operatorname{Cov}(\xi f(\xi) m, R) \quad \text { and } \quad R_{f}=\frac{1}{\mathbb{E}^{\mathbb{P}}[\xi f(\xi) m]},
$$

where we have used results in Section 3 and where $\xi$ is the worst-case distortion given by (14) so that $\xi f(\xi) m$ is the pricing kernel under model uncertainty.

We use the following annual U.S. consumption data for the period 1891-1998 (107 years) from Campbell [2003]:

$$
\mu_{c}=1.76 \%, \quad \sigma_{c}=3.218 \%, \quad \sigma_{S}=18.599 \% .
$$

We compute the probabilities of the three states occuring by requiring that they sum to 1 and match the mean $\mu_{c}$ and volatility $\sigma_{c}$ of consumption growth which gives probabilities of approximately $0.3269,0.3331$ and 0.3400 . Using (C.1) and (C.2), we compute the standard deviations of $m$ and $\xi f(\xi) m$, denoted $\sigma(m)$ and $\sigma(\xi f(\xi) m)$, as well as $R_{f}^{\mathrm{NoMU}}-1, R_{f}-1$, $E R^{\mathrm{NoMU}}$ and $E R$, all expressed as percentages, for different values of $\gamma$ and $\Omega$ and display the results in Table C.1, where we set $\bar{\beta}=0.9975$.

We see that the presence of model uncertainty lowers the risk-free rate, increases the excess return on the stock and increases the standard deviation of the pricing kernel (thus raising the Hansen and Jagannathan [1991] bound) relative to its absence.

For comparison with Table C.1, Campbell [2003] gives historical values for the U.S. for the period 1891-1998 of $R_{f}-1=2.020 \%$ and $E R=7.169 \%$ - the latter being the excess return on the U.S. equity market. We compute numerically the values of $\gamma$ and $\Omega$ which match these historical values - now setting $\bar{\beta}=1$ - and obtain

$$
\gamma=2.14, \quad \text { and } \quad \Omega=9.63
$$


Table C.2: Estimates of $\Omega$ for different error detection probabilities (EDP)

\begin{tabular}{rrrrrrrrr}
\hline EDP & 20 & 20 & 7 & 7 & 6 & 6 & 5 & 5 \\
\hline Distribution & LN & $3 \mathrm{~S}$ & LN & $3 \mathrm{~S}$ & LN & $3 \mathrm{~S}$ & $\mathrm{LN}$ & $3 \mathrm{~S}$ \\
$\Omega$ & 6.28 & 5.89 & 9.71 & 9.15 & 10.15 & 9.70 & 10.62 & 10.26 \\
\hline
\end{tabular}

Table C.3: Excess returns and risk-free rates for values of $\Omega$ consistent with $20 \%$ error detection probabilities

\begin{tabular}{rrrrrrrr}
\hline$\gamma$ & $\Omega$ & $\sigma(m)$ & $\sigma(\xi f(\xi) m)$ & $R_{f}^{\text {NoMU }}-1$ & $R_{f}-1$ & $E R^{\text {NoMU }}$ & $E R$ \\
\hline 2.14 & 5.89 & 6.63 & 20.95 & 3.47 & 2.70 & 1.42 & 4.46 \\
2.14 & 6.28 & 6.63 & 22.28 & 3.47 & 2.63 & 1.42 & 4.74 \\
2.14 & 9.63 & 6.63 & 33.89 & 3.47 & 2.02 & 1.42 & 7.17 \\
\hline
\end{tabular}

We observe that the value of the risk aversion parameter $\gamma$ is not implausibly high - indeed it is well within the range of 1 to 5 which is usually (Barillas et al. [2009], Maenhout [2004] or chapter 21, Cochrane [2005]) considered reasonable. But what about the value of $\Omega$ ? To estimate $\Omega$, we simulate consumption data consistent with the parameters in equation (C.3) for a time period spanning 107 years. To do this, we make two possible distributional assumptions concerning consumption growth. In the first (labelled LN), we assume consumption growth is log-normally distributed and in the second (labelled 3S), we assume it can take on the three values above with probabilities $0.3269,0.3331$ and 0.3400 which match $\mu_{c}$ and $\sigma_{c}$ and sum to 1 . We estimate $\Omega$ for four different error detection probabilities (labelled EDP) namely $20 \%, 7 \%, 6 \%$ and $5 \%$ - using the error detection probability methodology described in Section 5. The results are in Table C.2.

We see that the value of $\Omega=9.63$ in equation (C.4) estimated to match the historical values of $R_{f}-1=2.020 \%$ and $E R=7.169 \%$ is consistent with error detection probabilities in the region of $6 \%$ or $7 \%$. These error detection probabilities probabilities are low but not implausible and are, in fact, very close to those reported in Barillas et al. [2009] (p2407).

We recompute the quantities in Table C.1 with the values of $\Omega$ (6.28 for LN, 5.89 for 3S) corresponding to error detection probabilities of $20 \%$ as well as the value of $\Omega$ in equation (C.4) matching the Campbell [2003] data-set, setting $\bar{\beta}=1$ and $\gamma=2.14$, and report the results in Table C.3.

We see that error detection probabilities of $20 \%$ allow us to approximately triple the Hansen and Jagannathan [1991] bound $\sigma(m) / \mathbb{E}^{\mathbb{P}}[m]$ applicable in the absence of model uncertainty and takes $\sigma(\xi f(\xi) m) / \mathbb{E}^{\mathbb{P}}[\xi f(\xi) m]$ to around two-thirds of the value required to match the historical excess return of $7.169 \%$. Hence, we find that, similarly to Barillas et al. [2009] and Maenhout [2004], model uncertainty can potentially explain the equity premium puzzle of Mehra and Prescott [1985] - at least in part and possibly in whole - without increasing the risk-free rate and without implausibly high values of risk aversion $\gamma$. 
Table 1: Aversion to model uncertainty

\begin{tabular}{rrrrrr}
\hline Length of historical data-set & 500 & 1000 & 2000 & 4000 & 8000 \\
Value of $\Omega$ & 1.83 & 1.64 & 1.51 & 1.41 & 1.33 \\
\hline
\end{tabular}

Notes: Degree of aversion to model uncertainty as a function of the number of historical observations. $\Omega$ is estimated using the procedure in Section 5 , with 65000 bootstrapped samples, setting the confidence level to $80 \%$. The basis assets are taken to be a risk-free and a defaultable bond.

Table 2: Good deal bounds on Arrow-Debreu securities

\begin{tabular}{|c|c|c|c|c|c|c|c|c|}
\hline \multicolumn{9}{|c|}{$F_{\text {good }}$} \\
\hline $\bar{\Omega}$ & 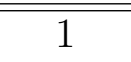 & 1.33 & $\bar{~} 1.41$ & $\overline{1.51}$ & 1.64 & $\begin{array}{l}1.83 \\
\end{array}$ & 4 & 8 \\
\hline C & 0.7364 & 0.7334 & 0.7321 & 0.7305 & 0.7282 & 0.7248 & 0.6882 & $0.6667^{\star}$ \\
\hline$\overline{\bar{c}}$ & 0.7893 & 0.7918 & 0.7929 & 0.7944 & 0.7964 & 0.7997 & $0.8333^{\star}$ & $0.8333^{\star}$ \\
\hline \multicolumn{9}{|c|}{$F_{\text {poor }}$} \\
\hline$\Omega$ & 1 & 1.33 & 1.41 & 1.51 & 1.64 & 1.83 & 4 & 8 \\
\hline$\underline{C}$ & 0.0882 & 0.0831 & 0.0809 & 0.0780 & 0.0738 & 0.0672 & $0.0000^{\star}$ & $0.0000^{\star}$ \\
\hline$\overline{\bar{C}}$ & 0.1939 & 0.1999 & 0.2024 & 0.2057 & 0.2103 & 0.2171 & 0.2903 & $0.3333^{\star}$ \\
\hline \multicolumn{9}{|c|}{$F_{\text {armageddon }}$} \\
\hline$\overline{\bar{\Omega}}$ & $\overline{11}$ & $\bar{~} 1.33$ & $\overline{\overline{1.41}}$ & $\bar{~} 1.51$ & $\bar{~} 1.64$ & 1.8 & $\overline{\overline{4}}$ & 8 \\
\hline 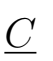 & 0.0697 & 0.0667 & 0.0655 & 0.0638 & 0.0615 & 0.0581 & 0.0215 & $0.0000^{\star}$ \\
\hline$\overline{\bar{C}}$ & 0.1226 & 0.1251 & 0.1262 & 0.1277 & 0.1298 & 0.1331 & $0.1667^{\star}$ & $0.1667^{\star}$ \\
\hline
\end{tabular}

Notes: The lower $\underline{C}$ and upper $\bar{C}$ good deal bounds as a function of the degree of uncertainty aversion $\Omega$ for the three Arrow-Debreu assets, $F_{\text {good }}, F_{\text {poor }}$ and $F_{\text {armageddon }}{ }^{\star}{ }$ means the good deal bound equals the relevant arbitrage bound. The basis assets are taken to be a risk-free and a defaultable bond.

Table 3: Optimal hedging portfolios

\begin{tabular}{rrrrrrrrr}
$\Omega$ & 1 & 1.25 & 1.5 & 1.75 & 2 & 4 & 8 \\
\hline & & & & & & & \\
$\underline{w_{1}}$ & 0.0697 & -1.1055 & -1.0678 & 0.0640 & 0.0596 & 0.0551 & 0.0215 & $0.0000^{\star}$ \\
$w_{2}$ & 1.8624 & 1.7992 & 1.6972 & 1.6168 & 1.5668 & 1.6011 & 0 \\
\hline & & & & & & & \\
$\bar{C}$ & 0.1226 & 0.1242 & 0.1275 & 0.1316 & 0.1361 & $0.1667^{\star}$ & $0.1667^{\star}$ \\
$w_{1}$ & -0.0483 & -0.0730 & -0.1070 & -0.1302 & -0.1454 & -0.8333 & -0.8333 \\
$w_{2}$ & -0.5163 & -0.4804 & -0.4377 & -0.4191 & -0.4175 & 1 & 1 \\
\hline
\end{tabular}

Notes: The optimal hedging position in the risk-free $\left(w_{1}\right)$ and the defaultable $\left(w_{2}\right)$ bond for the lower $\underline{C}$ and upper $\bar{C}$ good deal bounds as a function of the degree of uncertainty aversion $\Omega$. The focus asset is the Arrow-Debreu security that pays in the "armageddon" state, $F_{\text {armageddon. }}{ }^{\star}$ means the good deal bound equals the relevant arbitrage bound. The basis assets are taken to be a risk-free and a defaultable bond. 
Table 4: Optimal hedging portfolios (detailed)

\begin{tabular}{rrrrrrrrr}
\hline \hline$\Omega$ & 5 & 5.4 & 5.45 & 5.46 & 5.46125 & 5.4625 & 5.46375 & 5.465 \\
\hline$\underline{C}$ & 0.00655 & 0.00088 & 0.00019 & 0.00005 & 0.00003 & 0.00001 & $0.00000^{\star}$ & $0.00000^{\star}$ \\
$w_{1}$ & -0.7875 & -0.7832 & -0.7827 & -0.7826 & -0.7826 & -0.7826 & 0.0000 & 0.0000 \\
$w_{2}$ & 1.6799 & 1.7133 & 1.7175 & 1.7184 & 1.7185 & 1.7186 & 0.0000 & 0.0000 \\
\hline
\end{tabular}

Notes: The optimal hedging position in the risk-free $\left(w_{1}\right)$ and the defaultable $\left(w_{2}\right)$ bond for the lower $\underline{C}$ good deal bound as a function of the degree of uncertainty aversion $\Omega$. The focus asset is the ArrowDebreu security that pays in the "armageddon" state, $F_{\text {armageddon }}{ }^{\star}$ means the good deal bound equals the relevant arbitrage bound. The basis assets are taken to be a risk-free and a defaultable bond.

Table 5: Good deal bounds on options on the non-traded asset

\begin{tabular}{|c|c|c|c|c|c|c|c|c|c|}
\hline$\Omega$ & 16 & 8 & 4 & 2 & 1.75 & 1.5 & 1.25 & $\overline{1}$ & No MU \\
\hline \multicolumn{10}{|c|}{ European call, strike $K=65$} \\
\hline$\underline{C}$ & 0.000 & 0.058 & 0.738 & 2.601 & 3.051 & 3.560 & 4.092 & 4.445 & 4.445 \\
\hline $\bar{C}$ & 69.974 & 47.643 & 31.606 & 21.580 & 20.275 & 19.035 & 17.983 & 17.471 & 17.471 \\
\hline \multicolumn{4}{|c|}{ Risk-neutral price (same tree) } & & 8.937 & & BS & 8.917 & \\
\hline
\end{tabular}

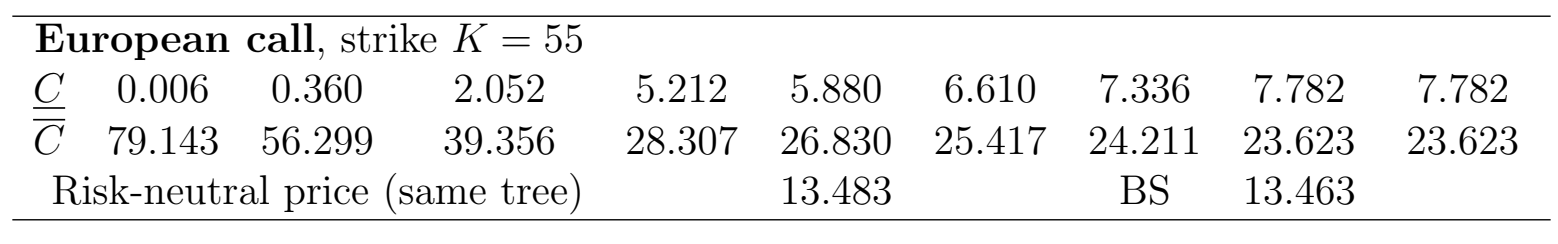

\begin{tabular}{|c|c|c|c|c|c|c|c|c|}
\hline \multicolumn{9}{|c|}{ European put, strike $K=65$} \\
\hline$\underline{C} \quad 0.001$ & 0.188 & 1.422 & 3.738 & 4.196 & 4.674 & 5.117 & 5.350 & 5.350 \\
\hline $\bar{C} \quad 33.503$ & 27.507 & 21.406 & 16.466 & 15.753 & 15.068 & 14.495 & 14.229 & 14.229 \\
\hline \multicolumn{4}{|c|}{ Risk-neutral price (same tree) } & 10.151 & & BS & 10.132 & \\
\hline
\end{tabular}

\begin{tabular}{|c|c|c|c|c|c|c|c|c|c|}
\hline$\Omega$ & 16 & 8 & 4 & 2 & 1.75 & 1.5 & 1.25 & 1 & No MU \\
\hline \multicolumn{10}{|c|}{ American call, strike $K=65$} \\
\hline$\underline{C}$ & 0.000 & 0.234 & 1.295 & 3.153 & 3.555 & 4.006 & 4.481 & 4.809 & 4.809 \\
\hline \multicolumn{5}{|c|}{ Risk-neutral price (same tree) } & 8.937 & & & & \\
\hline
\end{tabular}

\begin{tabular}{llllllll}
\hline American call, strike $K=55$ & & & & & & \\
$\underline{C} \quad 5.000 \quad 5.000 \quad 5.000$ & 6.927 & 7.360 & 7.844 & 8.355 & 8.695 & 8.695 \\
\hline Risk-neutral price (same tree) & & 13.483 & & & & \\
\hline
\end{tabular}

\begin{tabular}{llllllll}
\hline American put, strike $K=65$ & & & & & & \\
$\underline{C} 5.000 \quad 5.000 \quad 5.000$ & 6.162 & 6.475 & 6.803 & 7.114 & 7.284 & 7.284 \\
Risk-neutral price (same tree) & & 10.671 & & & & \\
\hline
\end{tabular}

Notes: The lower $\underline{C}$ and upper $\bar{C}$ good deal bounds on 2 year options on the non-traded asset as a function of the degree of uncertainty aversion $\Omega$. The basis assets are the traded asset and a one period risk-free bond, with hedging rebalancing possible every quarter. "BS" refers to the Black and Scholes [1973] price of the same option. For American options, only the lower good deal bounds $\underline{C}$ are shown. 